This item was submitted to Loughborough's Research Repository by the author.

Items in Figshare are protected by copyright, with all rights reserved, unless otherwise indicated.

\title{
Interaction problems between cracks and crystal defects in constrained Cosserat elasticity
}

PLEASE CITE THE PUBLISHED VERSION

https://doi.org/10.1142/S2424913018400118

\section{PUBLISHER}

(c) World Scientific Publishing Company

\section{VERSION}

AM (Accepted Manuscript)

\section{PUBLISHER STATEMENT}

Electronic version of an article published as Journal of Micromechanics and Molecular Physics, 3, 03n04, 2019, 184001, https://doi.org/10.1142/S2424913018400118 @ [copyright World Scientific Publishing Company] https://www.worldscientific.com/worldscinet/jmmp.

\section{LICENCE}

CC BY-NC-ND 4.0

\section{REPOSITORY RECORD}

Baxevanakis, Konstantinos, and H.G. Georgiadis. 2019. "Interaction Problems Between Cracks and Crystal Defects in Constrained Cosserat Elasticity”. figshare. https://hdl.handle.net/2134/36231. 


\title{
Interaction problems between cracks and crystal defects in constrained Cosserat elasticity
}

\author{
K.P. Baxevanakis ${ }^{1 *}$ and H.G. Georgiadis ${ }^{2,3}$ \\ ${ }^{1}$ Wolfson School of Mechanical, Electrical and Manufacturing Engineering, \\ Loughborough University, LE11 3TU, UK \\ ${ }^{2}$ Mechanics Division, National Technical University of Athens, Zographou, GR-15773, Greece \\ ${ }^{3}$ Office of Theoretical and Applied Mechanics, Academy of Athens, Greece
}

\begin{abstract}
In this work interaction problems between a finite-length crack with plane and antiplane crystal defects in the context of couple-stress elasticity are presented. Two alternative yet equivalent approaches for the formulation of crack problems are discussed based on the distributed dislocation technique. To this aim, the stress fields of climb and screw dislocation dipoles are derived within couple-stress theory and new 'constrained' rotational defects are introduced to satisfy the boundary conditions of the opening mode problem. Eventually, all interaction problems are described by single or systems of singular integral equations that are solved numerically using appropriate collocation techniques. The obtained results aim to highlight the deviation from classical elasticity solutions and underline the differences in interactions of cracks with single dislocations and dislocation dipoles. In general, it is concluded that the cracked body behaves in a more rigid way when couple-stresses are considered. Also, the stress level is significantly higher than the classical elasticity prediction. Moreover, the configurational forces acting on the defects are evaluated and their dependence on the characteristic material length of couple-stress theory and the distance between the defect and the crack-tip is discussed. This investigation reveals either a strengthening or a weakening effect in the opening mode problem while in the antiplane mode a strengthening effect is always obtained.
\end{abstract}

Keywords: Crack; Dislocation; Disclination; Dislocation Dipole; Microstructure; Couple-stress Elasticity; Cosserat Elasticity; Hyper-Singular Integral Equation; Distributed Dislocation Technique; Peach-Koehler Force.

\footnotetext{
*Corresponding author. Tel.: +44 (0) 1509 227030; fax: +44 (0) 1509227648.

E-mail address: K.Baxevanakis@lboro.ac.uk (K.P. Baxevanakis)
} 


\section{Introduction}

It is widely accepted that the macroscopically observed mechanical behavior of metal alloys is considerably affected by phenomena that occur in the microscale. Perhaps the most characteristic example of this connection is the phenomenon of crack initiation and growth which is followed by damage formation around the main crack. This damage has the form of microcracking and dislocation emission, manifested as discrete dislocations (monopoles) or dipoles Huang et al. [2006]. In fact, dislocation dipoles are found in much higher densities than single dislocations during plastic deformation [Gilman, 1964]. Therefore, interaction problems between cracks and crystal defects have been studied with a variety of analytical and experimental techniques over the past decades. For instance, Rice and Thomson [1974] proposed an energy condition for dislocation emission from a crack-tip and discussed the potential ways of subsequent fracture. Then, Thomson [1978] and Weertman [1978] introduced the idea of a dislocation shielded crack and the notion of the dislocation free zone. In this concept, the emitted dislocation is expected to glide away from the crack-tip until the interaction force between them is equilibrated by the lattice friction force so that the defect comes to rest. In addition, there are numerous experimental observations of these phenomena and we may refer indicatively to Kobayashi and Ohr [1980], Majumdar and Burns [1981], and Thomson [1987].

In this work, we extend our recent studies on interaction problems between cracks and dislocations in the framework of compatible couple-stress elasticity [Baxevanakis et al., 2017a; Baxevanakis et al., 2017b] offering alternative formulations for crack problems and investigating interactions between finite-length cracks and dipoles of dislocations. The problems are studied in the context of couple-stress elasticity (also known as constrained Cosserat theory), which a particular case of the general approach of Mindlin [1964] and is the simplest theory of elasticity in which couple-stresses are introduced. The fundamental concepts of couple-stress theory were first presented by Cosserat and Cosserat [1909], but the subject was generalized and reached maturity in the 1960s through the works of Mindlin and Tiersten [1962], Toupin [1962], and Koiter [1964]. It should be noted that the compatible couple-stress theory used herein was employed by other researchers to study crack problems under remote loading [see e.g. Gourgiotis and Georgiadis, 2008; Sternberg and Muki, 1967]. Under this framewowk, stress and strain 
quantities are successfully recovered. On the other hand, there is the possibility to use an incompatible formulation either in couple-stress elasticity or in other generalized continua, such as nonlocal and gradient elasticity [Mousavi, 2016; Mousavi and Lazar, 2015]. Employing this approach will provide an understanding of phenomena related to plasticity by offering information for quantities such as dislocation density fields.

A few solutions are reported on the interaction problems under consideration in the context of classical isotropic elasticity, however, these have not extensively investigated geometrical configurations and material combinations. Specifically, Zhang and Li [1991b] employed the complex potential method to calculate the stress intensity factors at the crack-tips and the image forces due to the presence of a discrete dislocation. Comninou [1987] examined a general configuration with the defect being out of the crack plane and discussed the associated closure effects. Then, Markenscoff [1993] provided a solution for the stress field ahead of the crack-tip using integral equations. Looking at dislocation pairs, Ballarini and Denda [1988] used the complex potential method to derive the stress intensity factors at the tips of a finite-length crack due to the interaction with a plane dislocation dipole of random orientation. The corresponding antiplane problem was studied by Lin et al. [1993]. In addition, Wang and Lee [1992]; [1993] proposed a criterion for emission of dipoles from a semi-infinite crack and identified the equilibrium states of a dislocation dipole near its crack-tip.

The formulation of crack problems in this work is based on the distributed dislocation technique (DDT), which proved a very efficient method for the analysis of crack problems in couple-stress elasticity. The main advantage of the method is that it provides full-field solutions at the expense of little analytical demands while requiring a relatively small computational cost compared to the Finite Element or Boundary Element methods. It should be remarked that the term 'distributed dislocations' does not constrain the technique to the use of single dislocations only. Indeed, any appropriate 'strain nucleus' capable of generating a traction-free crack when distributed along its faces may be used. In view of the above, we expand the use of the DDT in couple-stress elasticity by introducing dislocation dipoles as nuclei of strain in order to formulate the interaction problems. It is reminded that a dislocation dipole is defined as a pair of parallel dislocations that have equal and opposite sign Burgers vectors and are separated by a distance. Dislocation dipoles are area defects of the crystal lattice contrary to discrete dislocations that 
are line defects. Depending on their separation distance, dislocation dipoles can be identified as finite or infinitesimal [Kroupa, 1965], which are used as strain nuclei in crack problems. From a mathematical viewpoint, if the distributed defect is a discrete dislocation, the crack problem is formulated based on the gradient of the displacement field, which appears in the kernels of the governing integral equations. Accordingly, if dislocation dipoles are employed, the density of the defects corresponds to the crack face relative displacement. Hence, the first formulation is termed as slope formulation and the latter as displacement formulation. The main difference between the two approaches is that the displacement-based approach yields hyper-singular integral equations which may be more challenging to solve. On the other hand, this method is less computationally expensive since the displacement profile is obtained directly contrary to the slope method that requires an extra integration step. In fact, the displacement function is continuous across the crack length while the slope becomes unbounded at the crack-tips. Further, Chan et al. [2001] claim that this formulation offers alternative and simpler asymptotics of the integral equations kernels while Korsunsky and Hills [1995] proved that fewer terms are required in the displacement-based method to achieve the same level of accuracy. Other notable comparative works on these methods are provided by Korsunsky and Hills [1996], Dai [2002] and in the treatise by Hills et al. [1996].

The present study focuses on finite-length crack interactions with discrete climb and screw dislocations as well as with climb and screw dislocation dipoles. In all cases, the defects are placed along the crack plane so as not to induce crack closure effects. The crack problems are presented in parallel in each section of the paper. It is interesting to note that the satisfaction of the boundary conditions in the opening mode problems is not feasible by a distribution of a single translational defect. Indeed, rotational defects must be distributed across the crack length and these are termed as 'constrained' wedge disclination and infinitesimal 'constrained' wedge disclination dipole. Eventually, the governing singular and hyper-singular integral equations are solved numerically. One of the most interesting points in this work is the calculation of the configurational forces that act on the defects (J-integral and Peach-Koehler force). This investigation reveals an 'alternating' behavior between strengthening and weakening effects when the material microstructure is considered, depending on the distance of the defect from the cracktip and the ratio of the characteristic material length over the crack length. 


\section{Basic equations of couple-stress elasticity in plane and antiplane strain}

In this section, we briefly provide the basic equations of the equilibrium theory of plane and antiplane strain within the linearized couple-stress theory of homogeneous and isotropic elastic solids. The couple-stress elasticity theory (also known as constrained Cosserat theory) is the simplest theory of the so-called generalized continuum theories in which couple-stresses are introduced. For an overview of the basic concepts of linear couple-stress elasticity we refer to the fundamental papers of Toupin [1962], Mindlin and Tiersten [1962] and Koiter [1964].

\subsection{Plane strain}

The two-dimensional displacement field of a body that occupies a domain in the $(x, y)$-plane under plane strain conditions is described as

$$
u_{x} \equiv u_{x}(x, y) \neq 0, \quad u_{y} \equiv u_{y}(x, y) \neq 0, \quad u_{z} \equiv 0
$$

where the $z$ axis is perpendicular to the $(x, y)$-plane.

For the kinematical description of the elastic body, the following expressions are defined for the strain tensor, the rotation vector, and the curvature tensor components

$$
\begin{aligned}
& \varepsilon_{x x}=\frac{\partial u_{x}}{\partial x}, \quad \varepsilon_{x y}=\varepsilon_{y x}=\frac{1}{2}\left(\frac{\partial u_{x}}{\partial y}+\frac{\partial u_{y}}{\partial x}\right), \quad \varepsilon_{y y}=\frac{\partial u_{y}}{\partial y} \\
& \omega_{z} \equiv \omega=\frac{1}{2}\left(\frac{\partial u_{y}}{\partial x}-\frac{\partial u_{x}}{\partial y}\right), \\
& \kappa_{x z}=\frac{\partial \omega}{\partial x}, \quad \kappa_{y z}=\frac{\partial \omega}{\partial y} .
\end{aligned}
$$

From Eq. (3), it can be deduced that the rotation vector $\omega_{i}$ in couple-stress elasticity is not independent of the displacement vector $u_{i}$ since the normal component of the rotation is fully described by the distribution of the tangential displacements over the boundary. 
Next, the expressions of force and moment equilibrium in the absence of body forces and body couples take the form

$$
\frac{\partial \sigma_{x x}}{\partial x}+\frac{\partial \sigma_{y x}}{\partial y}=0, \quad \frac{\partial \sigma_{x y}}{\partial x}+\frac{\partial \sigma_{y y}}{\partial y}=0, \quad \sigma_{x y}-\sigma_{y x}+\frac{\partial m_{x z}}{\partial x}+\frac{\partial m_{y z}}{\partial y}=0,
$$

where $\sigma_{p q}$ and $m_{p q}$ are the components of the stress tensor and couple-stress tensor, which are both asymmetric.

Assuming a linear and isotropic material response the strain energy density takes the following form

$$
W=(\lambda / 2)\left(\varepsilon_{x x}+\varepsilon_{y y}\right)^{2}+\mu\left(\varepsilon_{x x}^{2}+2 \varepsilon_{x y}^{2}+\varepsilon_{y y}^{2}\right)+2 \mu \ell^{2}\left(\kappa_{x z}^{2}+\kappa_{y z}^{2}\right)
$$

where $\lambda$ and $\mu$ are Lamé type constants and $\ell$ is the characteristic length introduced in couplestress elasticity [Mindlin, 1963].

Then, the constitutive equations in the plane-strain case become

$$
\begin{aligned}
& \varepsilon_{x x}=(2 \mu)^{-1}\left[\sigma_{x x}-\nu\left(\sigma_{x x}+\sigma_{y y}\right)\right], \quad \varepsilon_{y y}=(2 \mu)^{-1}\left[\sigma_{y y}-\nu\left(\sigma_{x x}+\sigma_{y y}\right)\right] \\
& \varepsilon_{x y}=(4 \mu)^{-1}\left(\sigma_{x y}+\sigma_{y x}\right)
\end{aligned}
$$

and

$$
\kappa_{x z}=\left(4 \mu \ell^{2}\right)^{-1} m_{x z}, \quad \kappa_{y z}=\left(4 \mu \ell^{2}\right)^{-1} m_{y z} .
$$

In view of the above, the non-vanishing components of the asymmetric stress tensor $\sigma_{p q}$ in terms of the displacement components are given as 


$$
\begin{aligned}
\sigma_{x x} & =(\lambda+2 \mu) \frac{\partial u_{x}}{\partial x}+\lambda \frac{\partial u_{y}}{\partial y}, \quad \sigma_{y y}=(\lambda+2 \mu) \frac{\partial u_{y}}{\partial y}+\lambda \frac{\partial u_{x}}{\partial x} \\
\sigma_{y x} & =\mu\left(\frac{\partial u_{x}}{\partial y}+\frac{\partial u_{y}}{\partial x}\right)+\mu \ell^{2}\left(\frac{\partial^{3} u_{y}}{\partial x^{3}}-\frac{\partial^{3} u_{x}}{\partial x^{2} \partial y}+\frac{\partial^{3} u_{y}}{\partial x \partial y^{2}}-\frac{\partial^{3} u_{x}}{\partial y^{3}}\right), \\
\sigma_{x y} & =\mu\left(\frac{\partial u_{x}}{\partial y}+\frac{\partial u_{y}}{\partial x}\right)-\mu \ell^{2}\left(\frac{\partial^{3} u_{y}}{\partial x^{3}}-\frac{\partial^{3} u_{x}}{\partial x^{2} \partial y}+\frac{\partial^{3} u_{y}}{\partial x \partial y^{2}}-\frac{\partial^{3} u_{x}}{\partial y^{3}}\right) .
\end{aligned}
$$

\subsection{Antiplane strain}

We now consider a body that occupies a domain in the $(x, y)$-plane under antiplane strain conditions. In this case, the displacement field is written as

$$
u_{x}=u_{y}=0, \quad u_{z} \equiv w \neq 0, \quad w \equiv w(x, y)
$$

The non-vanishing components of the strain tensor, the rotation vector, and the curvature tensor are defined as

$$
\begin{aligned}
& \varepsilon_{x z}=\frac{1}{2} \frac{\partial w}{\partial x}, \quad \varepsilon_{y z}=\frac{1}{2} \frac{\partial w}{\partial y}, \quad \omega_{x}=\frac{1}{2} \frac{\partial w}{\partial y}, \quad \omega_{y}=-\frac{1}{2} \frac{\partial w}{\partial x}, \\
& \kappa_{x x}=-\kappa_{y y}=\frac{1}{2} \frac{\partial^{2} w}{\partial x \partial y}, \quad \kappa_{x y}=-\frac{1}{2} \frac{\partial^{2} w}{\partial x^{2}}, \quad \kappa_{y x}=\frac{1}{2} \frac{\partial^{2} w}{\partial y^{2}} .
\end{aligned}
$$

The strain energy density in the case of a linear and isotropic material response takes the following form

$$
W=2 \mu\left(\varepsilon_{x z}^{2}+\varepsilon_{y z}^{2}\right)+2\left(\eta+\eta^{\prime}\right)\left(\kappa_{x x}^{2}+\kappa_{y y}^{2}\right)+2 \eta\left(\kappa_{x y}^{2}+\kappa_{y x}^{2}\right)+4 \eta^{\prime} \kappa_{x y} \kappa_{y x},
$$

where $\mu$ has the same meaning as the shear modulus in the classical theory, and $\left(\eta, \eta^{\prime}\right)$ are the couple-stress moduli with dimensions of [ force]. The elastic moduli must satisfy the following inequalities so that the strain energy density is positive definite 
$\mu>0, \quad \eta>0, \quad-1<\beta=\eta^{\prime} / \eta<1$.

Further, the stress and couple-stress components are written in terms of the displacement field as

$$
\begin{aligned}
& \sigma_{x z}=\mu \frac{\partial}{\partial x}\left(w-\ell^{2} \nabla^{2} w\right), \quad \sigma_{z x}=\mu \frac{\partial}{\partial x}\left(w+\ell^{2} \nabla^{2} w\right), \\
& \sigma_{y z}=\mu \frac{\partial}{\partial y}\left(w-\ell^{2} \nabla^{2} w\right), \quad \sigma_{z y}=\mu \frac{\partial}{\partial y}\left(w+\ell^{2} \nabla^{2} w\right) . \\
& m_{x x}=4\left(\eta+\eta^{\prime}\right) \kappa_{x x}=2\left(\eta+\eta^{\prime}\right) \frac{\partial^{2} w}{\partial x \partial y} \\
& m_{y y}=4\left(\eta+\eta^{\prime}\right) \kappa_{y y}=-2\left(\eta+\eta^{\prime}\right) \frac{\partial^{2} w}{\partial x \partial y}=-m_{x x}, \\
& m_{x y}=4 \eta \kappa_{x y}+4 \eta^{\prime} \kappa_{y x}=-2 \eta \frac{\partial^{2} w}{\partial x^{2}}+2 \eta^{\prime} \frac{\partial^{2} w}{\partial y^{2}} \\
& m_{y x}=4 \eta \kappa_{y x}+4 \eta^{\prime} \kappa_{x y}=2 \eta \frac{\partial^{2} w}{\partial y^{2}}-2 \eta^{\prime} \frac{\partial^{2} w}{\partial x^{2}}
\end{aligned}
$$

where $\ell=(\eta / \mu)^{1 / 2}$ is the characteristic material length of isotropic couple-stress elasticity.

We also cite at this point the pertinent tractions that can be prescribed on a surface defined by the unit normal $\mathbf{n}=(0, \pm 1)$ [Mindlin and Tiersten, 1962]

$$
P_{z}^{(n)} \equiv t_{y z}=\sigma_{y z}+\frac{1}{2} \frac{\partial m_{y y}}{\partial x}, \quad R_{x}^{(n)}=m_{y x}
$$

where $t_{y z}$ denotes the total shear stress. These expressions will be useful in the formulation of the antiplane crack problem.

\section{Formulation of the interaction problems}

\subsection{Opening mode interaction problem}

In this section we describe the formulation of crack problems based on the distributed dislocation technique. In Fig. 1, the two alternative approaches for the description of two-dimensional crack 
problems are schematically depicted. If the crack is formulated based on a continuous distribution of discrete dislocations, the crack opening profile may be represented by a pile of narrow strips that correspond to climb dislocations [Dai, 2002]. On the contrary, if a continuous distribution of infinitesimal dislocation dipoles is employed, the crack opening displacement is formed by an array of parallel thin strips, corresponding to infinitesimal dislocation dipoles. In the first case, the normal crack face displacement at any point along the crack faces is the sum of Burgers vectors of all dislocations at the same point while in the latter case the crack opening displacement is equal to the Burgers vector of each dislocation dipole at the same location. In both cases, the distributed defects should not be misinterpreted as crystallographic defects but solely as a method to create a traction-free crack.

In all crack problems investigated herein, we consider a straight crack of finite-length $2 a$ in an infinite elastic microstructured domain characterized by couple-stress elasticity theory. In this section, we study the opening mode interaction problem and therefore the crack is assumed to interact with a discrete climb dislocation $b_{y}$ or with a horizontal climb dislocation dipole $b_{y y}$ lying along the crack plane $(y=0)$ at a distance $d$ from the crack center, as described in Fig. 1. Plane strain conditions prevail, and no other remote loading is applied to the body. The crack faces are described by the outward normal unit vector $\mathbf{n}=(0, \pm 1)$ and are assumed to be traction-free. Hence, the boundary conditions along the crack faces take the following form

$$
\sigma_{y x}(x, 0)=0, \quad \sigma_{y y}(x, 0)=0, \quad m_{y z}=0, \quad \text { for } \quad|x|<a,
$$

augmented by the regularity conditions at infinity

$$
\sigma_{p q}^{\infty} \rightarrow 0, \quad m_{q z}^{\infty} \rightarrow 0 \quad \text { as } \quad r \rightarrow \infty
$$

where $(p, q)=(x, y)$ and $r=\left(x^{2}+y^{2}\right)^{1 / 2}$ is the distance from the origin. 


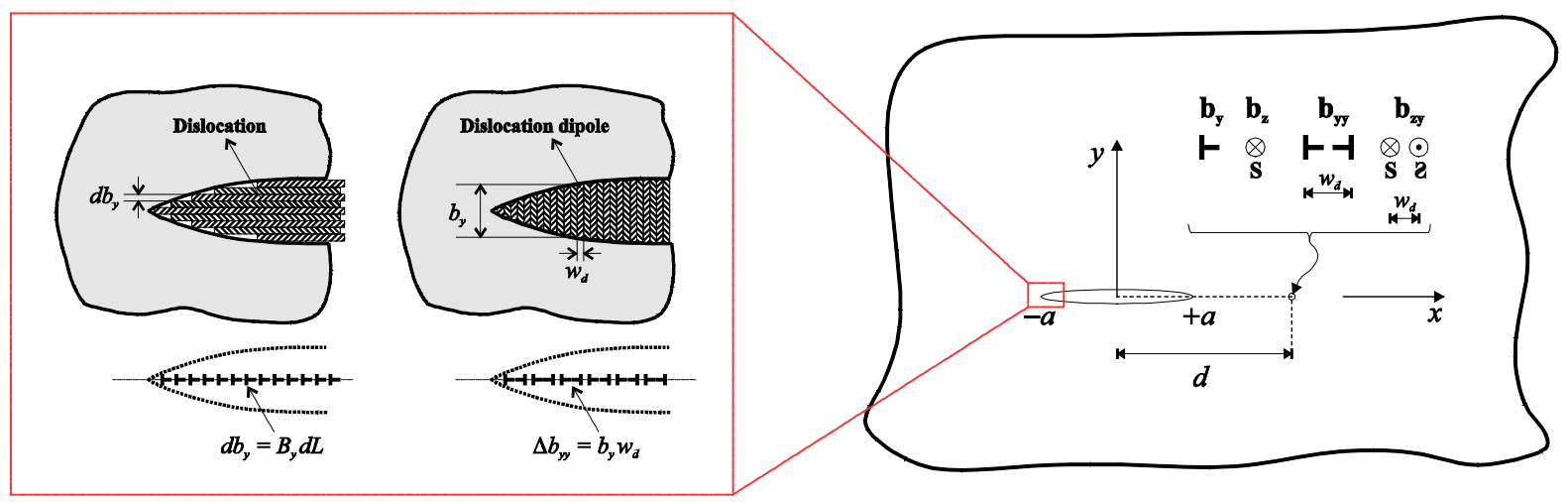

Fig. 1: Geometry of the interaction problem between a finite-length plane crack and different crystal defects. The detail depicts the two alternative formulations of the opening mode crack problem.

It is noted that a discrete climb dislocation in an infinite isotropic couple-stress domain induces both normal stresses $\sigma_{y y}^{\left(b_{y}\right)}(x, 0)$ and couple-stresses $m_{y z}^{\left(b_{y}\right)}(x, 0)$ along the slip plane $y=0$ [Baxevanakis et al., 2017a]. On the other hand, there are no shear stresses induced at $y=0$, so that $\sigma_{y x}^{\left(b_{y}\right)}(x, 0)=0$.

Using the full-field solutions for discrete dislocations in couple-stress elasticity, we may now derive the stress field expressions for dislocation dipoles. In fact, the types of translational dislocation met in this work (climb and screw) may be placed in pairs along the $x$ or $y$ axis to form two types of horizontal and two types of vertical dislocation dipoles (Fig. 2). All these configurations are appropriate for the formulation of crack problems (see Hills et al. [1996]). Cases (a) and (c) correspond to opening type of displacement discontinuity and cases (b) and (d) to antiplane deformation modes. Dislocation dipoles are usually characterised by their intensity $b_{i j}$, where $i$ denotes the direction of the Burgers vector of the two dislocations and $j$ is the normal direction to the distance $w_{d}$ that separates the dislocation pair.

Moreover, dislocation dipoles are distinguished to infinitesimal and finite based on the separation distance $w_{d}$ [Kroupa, 1965]. The stress fields of a finite dislocation dipole are derived by superposing the corresponding fields of the two discrete dislocations that form the pair. Then, to create an infinitesimal dislocation dipole, it is required that $w_{d} \rightarrow 0$ and $b \rightarrow \infty$ while the quantity $w_{d} b$ is finite [Weertman, 1996]. In fact, the stress field of an infinitesimal dislocation dipole may be derived by differentiation of the field of the constituent discrete dislocation. 


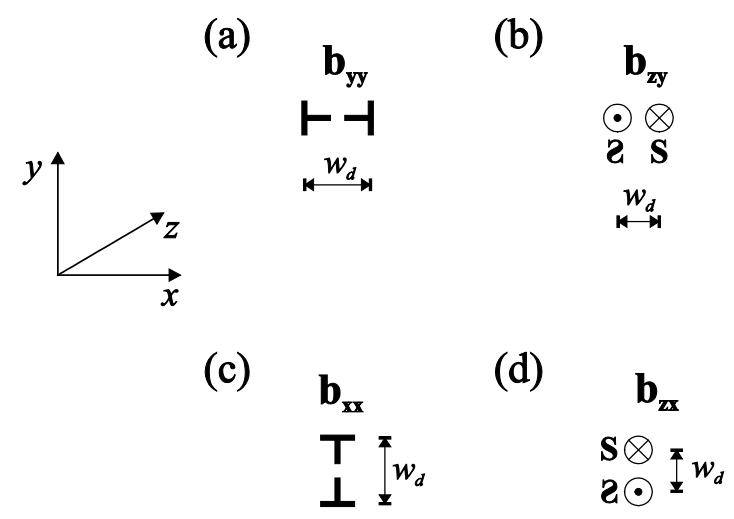

Fig. 2: Horizontal and vertical dislocation dipoles.

Following this procedure, we derive the stress and couple-stress expressions of an infinitesimal climb dislocation dipole, which will serve as influence functions in the opening mode crack problem. The components of interest (see Eq. (18)) read as

$$
\begin{aligned}
\sigma_{y y}^{\left(b_{y y}\right)}= & \frac{\mu b_{y} w_{d}}{2 \pi(1-\nu) r^{2}}(2 \cos 2 \theta-\cos 4 \theta)+\frac{6 \mu b_{y} w_{d}}{\pi r^{2}}\left[\frac{2 \ell^{2}}{r^{2}}-K_{2}(r / \ell)\right] \cos 4 \theta \\
& +\frac{\mu b_{y} w_{d}}{4 \pi \ell^{2}}\left\{K_{0}(r / \ell)+\left[3 K_{0}(r / \ell)-4 K_{2}(r / \ell)\right] \cos 4 \theta\right\} \\
\sigma_{y x}^{\left(b_{y y}\right)}= & -\frac{\mu b_{y} w_{d}}{2 \pi(1-\nu) r^{2}}(\sin 2 \theta-\sin 4 \theta)-\frac{6 \mu b_{y} w_{d}}{\pi r^{2}}\left[\frac{2 \ell^{2}}{r^{2}}-K_{2}(r / \ell)\right] \sin 4 \theta \\
& +\frac{\mu b_{y} w_{d}}{4 \pi \ell^{2}}\left\{2 K_{0}(r / \ell) \sin 2 \theta-\left[3 K_{0}(r / \ell)-4 K_{2}(r / \ell)\right] \sin 4 \theta\right\} \\
m_{y z}^{\left(b_{y y}\right)}= & -\frac{\mu b_{y} w_{d}}{4 \pi \ell^{2}} r\left[K_{2}(r / \ell)-K_{0}(r / \ell)\right](\cos \theta-\cos 3 \theta) \\
& -\frac{2 \mu b_{y} w_{d}}{\pi r}\left[\frac{2 \ell^{2}}{r^{2}}-K_{2}(r / \ell)\right] \cos 3 \theta .
\end{aligned}
$$

From Eqs. (20) and (22) we deduce that a climb dislocation dipole (either infinitesimal or finite) in an infinite isotropic couple-stress body induces both normal stresses $\sigma_{y y}^{\left(b_{y y}\right)}(x, 0)$ and couplestresses $m_{y z}^{\left(b_{y y}\right)}(x, 0)$ along the slip plane $(y=0)$ whereas there are no shear stresses produced by this defect at the slip plane, so that $\sigma_{y x}^{\left(b_{y y}\right)}(x, 0)=0$. Also, employing asymptotic analysis it 
may be shown that the stress components retain the quadratic singularity that arises in classical elasticity while the couple-stress $m_{y z}$ has a Cauchy type singularity. The full-field solution reduces to that of classical isotropic elasticity for $\ell \rightarrow 0$ [Weertman, 1996]. As it will be shown later, the stress fields of dislocation dipoles reduce faster with respect to the distance compared to that of discrete dislocations.

Returning to the crack problem under consideration, the solution strategy is based on the decomposition of the initial crack problem to two auxiliary problems and the superposition of their solutions. In the first auxiliary problem, we consider an uncracked body subjected to the loading of a discrete climb dislocation (or of a horizontal climb dislocation dipole) that is placed along the crack line $y=0$ and at a distance $d$ from the crack center. In the second auxiliary problem, a geometrically identical body to the initial cracked one without the crystal defect is studied. In this case, the loading consists of equal and opposite tractions to those generated in the first auxiliary problem and is applied along the crack faces. Hence, the boundary conditions along the crack faces $(|x|<a)$ take the following form

$$
\sigma_{y y}(x, 0)=-\sigma_{y y}^{c l . d .}(x-d, 0), \quad \sigma_{y x}(x, 0)=0, m_{y z}(x, 0)=-m_{y z}^{c l . d .}(x-d, 0),
$$

augmented with the regularity conditions (19). The superscript "cl.d." corresponds to either a discrete climb dislocation or a climb dislocation dipole. It is remarked that the same interaction problem in the context of classical isotropic elasticity is described by the first two conditions of Eq. (23). In that framework, a distribution of discrete climb dislocations or equivalently of infinitesimal horizontal climb dislocation dipoles would be sufficient [Hills et al., 1996]. However, in couple-stress elasticity it is not possible to simultaneously satisfy all three boundary conditions of Eq. (23) by a distribution of a single nucleus of strain (either discrete dislocations or dislocation dipoles) only. As discussed in Baxevanakis et al. [2017a], to satisfy the above boundary conditions, it is necessary to distribute along the crack faces discontinuities both in the displacement and in the rotation vector. The necessary rotational defect that together with a distribution of discrete climb dislocations satisfies the boundary conditions was termed as 'constrained' wedge disclination $\left(\Omega_{z}\right)$. Generalizing this concept, we introduce the infinitesimal 
'constrained' wedge disclination dipole $\left(\Omega_{z y}\right)$ as the necessary rotational defect to be distributed along the crack faces together with infinitesimal climb dislocation dipoles so that the boundary conditions of the problem are satisfied. This defect is produced by two constrained wedge disclinations of opposite sign with the distance between them approaching zero.

Then, the influence functions corresponding to the normal stress $\sigma_{y y}$ and to the couplestress $m_{y z}$ along the crack line $y=0$ are obtained from the superposition of the stress and couple-stress fields of the translational and rotational defects described above. In the case of distributed discrete defects, these read as

$$
\sigma_{y y}(x, 0)=\sigma_{y y}^{\left(b_{y}\right)}(x, 0)+\sigma_{y y}^{\left(\Omega_{z}\right)}(x, 0), \quad m_{y z}(x, 0)=m_{y z}^{\left(b_{y}\right)}(x, 0)+m_{y z}^{\left(\Omega_{z}\right)}(x, 0),
$$

where

$$
\begin{array}{ll}
\sigma_{y y}^{\left(b_{y}\right)}(x, 0)=b_{y} L_{11}(x), & \sigma_{y y}^{\left(\Omega_{z}\right)}(x, 0)=\Omega_{z} L_{12}(x), \\
m_{y z}^{\left(b_{y}\right)}(x, 0)=b_{y} L_{12}(x), & m_{y z}^{\left(\Omega_{z}\right)}(x, 0)=\Omega_{z} L_{22}(x),
\end{array}
$$

and

$$
\begin{aligned}
& L_{11}(x)=\frac{\mu}{2 \pi(1-\nu) x}+\frac{2 \mu}{\pi x}\left[\frac{2 \ell^{2}}{x^{2}}-K_{2}\left(\frac{|x|}{\ell}\right)\right], \\
& L_{12}(x)=\frac{\mu}{\pi}\left[K_{2}\left(\frac{|x|}{\ell}\right)-\frac{2 \ell^{2}}{x^{2}}\right]-\frac{\mu}{\pi} K_{0}\left(\frac{|x|)}{\ell}\right) \\
& L_{22}(x)=\frac{\mu \ell}{2 \pi} \operatorname{sgn}(x) G_{1,3}^{2,1}\left(\left.\frac{x^{2}}{4 \ell^{2}}\right|_{-1 / 2,1 / 2,0} ^{1}\right)
\end{aligned}
$$

where $K_{i}(|x| / \ell)$ is the $i^{\text {th }}$ order modified Bessel function of the second kind and $G_{c, d}^{a, b}()$ is the MeijerG function [Erdélyi et al., 1953].

Accordingly, if defect dipoles are used, the influence functions take the following form

$$
\sigma_{y y}(x, 0)=\sigma_{y y}^{\left(b_{y y}\right)}(x, 0)+\sigma_{y y}^{\left(\Omega_{z y}\right)}(x, 0), \quad m_{y z}(x, 0)=m_{y z}^{\left(b_{y y}\right)}(x, 0)+m_{y z}^{\left(\Omega_{z y}\right)}(x, 0),
$$


where

$$
\begin{aligned}
\sigma_{y y}^{\left(b_{y y}\right)}(x, 0) & =b_{y} w_{d}\left[L_{11}(x)+\left(L_{22}(x) / \ell^{2}\right)\right], & \sigma_{y y}^{\left(\Omega_{z y}\right)}(x, 0)=\Omega_{z} w_{d} L_{12}(x), \\
m_{y z}^{\left(b_{y y}\right)}(x, 0) & =b_{y} w_{d} L_{12}(x), & m_{y z}^{\left(\Omega_{z y}\right)}(x, 0)=\Omega_{z} w_{d} L_{22}(x),
\end{aligned}
$$

and

$$
\begin{aligned}
& L_{11}(x)=\frac{\mu}{2 \pi(1-\nu) x^{2}}+\frac{6 \mu}{\pi x^{2}}\left[\frac{2 \ell^{2}}{x^{2}}-K_{2}\left(\frac{|x|}{\ell}\right)\right], \quad L_{12}(x)=\frac{2 \mu}{\pi x}\left[K_{2}\left(\frac{|x|}{\ell}\right)-\frac{2 \ell^{2}}{x^{2}}\right], \\
& L_{22}(x)=\frac{\mu}{\pi}\left[K_{0}\left(\frac{|x|}{\ell}\right)-K_{2}\left(\frac{|x|}{\ell}\right)\right] .
\end{aligned}
$$

It is interesting to remark that no normal stresses are induced at infinity due to the rotational defects. Also, as $\ell \rightarrow 0$, the couple-stress $m_{y z}$ vanishes whereas the normal stress $\sigma_{y y}$ reduces to the corresponding influence function for the opening mode problem in classical elasticity, for both formulation approaches [Hills et al., 1996].

\subsection{Antiplane mode interaction problem}

The interaction problem of a finite-length crack with antiplane defects is discussed next. The geometrical configuration is the same as in the previous section: the crack length is $2 a$ and the crystal defect (discrete screw dislocation $b_{z}$ or horizontal screw dislocation dipole $b_{z y}$ ) lies at the crack plane at a distance $d$ from the crack center (Fig. 1) while there is no other loading to the body. Antiplane strain conditions prevail and the crack faces are assumed traction-free. In view of Eq. (17), the boundary conditions of this interaction problem are given as

$$
t_{y z}(x, 0)=\sigma_{y z}(x, 0)+\frac{1}{2} \partial_{x} m_{y y}(x, 0)=0, \quad m_{y x}=0, \quad \text { for } \quad|x|<a
$$

along with the regularity conditions at infinity 


$$
\sigma_{p z}^{\infty} \rightarrow 0, \quad m_{p q}^{\infty} \rightarrow 0 \quad \text { as } \quad r \rightarrow \infty
$$

where $(p, q)=(x, y)$ and $r=\left(x^{2}+y^{2}\right)^{1 / 2}$ is the distance from the origin.

As discussed in Baxevanakis et al. [2017b], a discrete screw dislocation in couple-stress elasticity generates only shear stresses $t_{y z}^{\left(b_{z}\right)}(x, 0)$ along the crack plane, so that $m_{y x}^{\left(b_{z}\right)}(x, 0)=0$. Following a similar procedure as in Section 3.1, we obtain the stress and couple-stress expressions of an infinitesimal screw dislocation dipole that will serve as influence functions in the antiplane shear mode crack problem as

$$
\begin{aligned}
& \sigma_{y z}^{\left(b_{z y}\right)}=\frac{\mu b_{z} w_{d}}{2 \pi r^{2}} \cos 2 \theta-\frac{3 \mu b_{z} \ell^{2} w_{d}(1+\beta)}{\pi r^{4}} \cos 4 \theta \\
& m_{y y}^{\left(b_{z y}\right)}=-m_{x x}^{\left(b_{z y}\right)}=\frac{2 \mu b_{z} \ell^{2} w_{d}(1+\beta)}{\pi r^{3}} \cos 3 \theta-\frac{12 \mu b_{z} \ell^{2} w_{d}(1+\beta)^{2}}{\pi r^{3}}\left[\frac{2 \ell^{2}}{r^{2}}-K_{2}(r / \ell)\right] \\
& \times \cos 5 \theta+\frac{\mu b_{z} w_{d}(1+\beta)^{2}}{4 \pi r}\left\{K_{2}(r / \ell) \cos 3 \theta-\left[6 K_{0}(r / \ell)-9 K_{2}(r / \ell)\right] \cos 5 \theta\right\} \\
& +\frac{\mu b_{z} w_{d}(1+\beta)^{2}}{32 \pi \ell^{2}} r\left[K_{0}(r / \ell)-K_{2}(r / \ell)\right](2 \cos \theta-\cos 3 \theta-\cos 5 \theta), \\
& m_{y x}^{\left(b_{z y}\right)}=-\frac{2 \mu b_{z} \ell^{2} w_{d}(1+\beta)}{\pi r^{3}} \sin 3 \theta+\frac{12 \mu b_{z} \ell^{2} w_{d}(1+\beta)^{2}}{\pi r^{3}}\left[\frac{2 \ell^{2}}{r^{2}}-K_{2}(r / \ell)\right] \sin 5 \theta \\
& +\frac{3 \mu b_{z} w_{d}(1+\beta)^{2}}{4 \pi r}\left[2 K_{0}(r / \ell)-3 K_{2}(r / \ell)\right] \sin 5 \theta+\frac{\mu b_{z} w_{d}(1+\beta)(1-3 \beta)}{4 \pi r} \\
& \times K_{2}(r / \ell) \sin 3 \theta-\frac{\mu b_{z} w_{d}\left(1-\beta^{2}\right)}{16 \pi \ell^{2}} r\left[K_{0}(r / \ell)-K_{2}(r / \ell)\right](\sin \theta+\sin 3 \theta) \\
& +\frac{\mu b_{z} w_{d}(1+\beta)^{2}}{32 \pi \ell^{2}} r\left[K_{0}(r / \ell)-K_{2}(r / \ell)\right](\sin 3 \theta+\sin 5 \theta) \text {. }
\end{aligned}
$$

Eqs. (32) and (33) show that for $y=0$ this defect generates shear stresses $\sigma_{y z}^{\left(b_{z y}\right)}(x, 0)$ and couple-stresses $m_{y y}^{\left(b_{z y}\right)}(x, 0)$ along the crack plane, while $m_{y x}^{\left(b_{z y}\right)}(x, 0)=0$. Moreover, based on asymptotic analysis it is deduced that the shear stresses exhibit an $r^{-4}$ singularity, whereas the 
couple-stresses behave as $r^{-3}$ at the dislocation core. For $\beta \rightarrow-1$, i.e. $\eta=-\eta^{\prime}$, the classical elasticity solution is recovered.

For the solution of the antiplane crack problem, we follow the same decomposition approach described earlier. In the first auxiliary problem, an uncracked geometrically identical to the initial body is considered that is subjected to the loading of a discrete screw dislocation (or of a horizontal screw dislocation dipole) lying along the crack line at a distance $d$ from the crack center. Then, the 'corrective solution' problem consists of a cracked body loaded along the crack faces with equal and opposite tractions to those generated in the first auxiliary problem. In this case, the boundary conditions are

$$
\begin{aligned}
& t_{y z}(x, 0)=-t_{y z}^{s c r . d .}(x-d, 0)=-\sigma_{y z}^{s c r . d .}(x-d, 0)-\frac{1}{2} \partial_{x} m_{y y}^{s c r . d .}(x-d, 0), \\
& m_{y x}^{s c r . d .}(x, 0)=0, \quad \text { for }|x|<a,
\end{aligned}
$$

supplemented by the regularity conditions (31). The superscript "scr.d." corresponds to either a discrete screw dislocation or a screw dislocation dipole. The boundary conditions in Eq. (35) are satisfied by either a distribution of discrete screw dislocations or by a distribution of infinitesimal horizontal screw dislocation dipoles along the crack faces. Eq. $(35)_{2}$ is automatically satisfied since neither of the antiplane defects induces couple-stresses $m_{y x}$ along the line $y=0$. In classical elasticity, a distribution of a single nucleus of strain would also be sufficient to satisfy the boundary condition of the problem (Eq. $\left.(35)_{1}\right)$.

Then, the influence function for the antiplane mode problem in the case of distributed discrete defects takes the form

$$
\begin{aligned}
& t_{y z}^{\left(b_{z}\right)}(x, 0)= \sigma_{y z}^{\left(b_{z}\right)}(x, 0)+\frac{1}{2} \partial_{x} m_{y y}^{\left(b_{z}\right)}(x, 0)= \\
&=\frac{\mu b_{z}}{2 \pi x}-\frac{2 \mu b \ell^{2}(1+\beta)}{\pi x^{3}}+\frac{6 \mu b_{z} \ell^{2}(1+\beta)^{2}}{\pi x^{3}}\left[\frac{2 \ell^{2}}{x^{2}}-K_{2}\left(\frac{|x|}{\ell}\right)\right] . \\
& \quad-\frac{\mu b_{z}(1+\beta)^{2}}{4 \pi x}\left[5 K_{2}\left(\frac{|x|)}{\ell}\right)-3 K_{0}\left(\frac{|x|}{\ell}\right)\right]
\end{aligned}
$$


It can be shown that the total shear stress given in Eq. (36) exhibits a cubic singularity $x^{-3}$ as $x \rightarrow 0$.

Additionally, the corresponding influence function in a displacement-based formulation framework is written as

$$
\begin{aligned}
t_{y z}^{\left(b_{z y}\right)}( & x, 0)=\sigma_{y z}^{\left(b_{z y}\right)}(x, 0)+\frac{1}{2} \partial_{x} m_{y y}^{\left(b_{z y}\right)}(x, 0)=\frac{\mu b_{z} w_{d}}{2 \pi x^{2}}-\frac{6 \mu b_{z} w_{d} \ell^{2}(1+\beta)}{\pi x^{4}} \\
& +\frac{30 \mu b_{z} w_{d} \ell^{2}(1+\beta)^{2}}{\pi x^{4}}\left[\frac{2 \ell^{2}}{x^{4}}-K_{2}(|x| / \ell)\right]-\frac{3 \mu b_{z} w_{d}(1+\beta)^{2}}{4 \pi x^{2}} \\
& \times\left[9 K_{2}(|x| / \ell)-5 K_{0}(|x| / \ell)\right]-\frac{\mu b_{z} w_{d}(1+\beta)^{2}}{4 \pi \ell^{2}}\left[K_{2}(|x| / \ell)-K_{0}(|x| / \ell)\right] .
\end{aligned}
$$

The previous expression behaves as $O\left(x^{-4}\right)$ as $x \rightarrow 0$. Also, it is worth noting that for $\beta=-1$, both Eqs. (36) and (37) reduce to the influence functions of classical elasticity [Bilby and Eshelby, 1968].

\section{Outline of solution}

\subsection{Opening mode interaction problem}

Based on the analysis in Section 3.1, the corrective stresses (Eq. (23)) are generated by a continuous distribution of discrete climb dislocations and constrained wedge disclinations or, equivalently, of climb dislocation dipoles and constrained wedge disclination dipoles along the crack faces. The elastic field generated by each distribution is obtained through integration of the corresponding influence functions along the crack faces, i.e. Eqs. (24)-(26) or Eqs. (27)-(29). Then, the simultaneous satisfaction of the first and third conditions of Eq. (23) leads to a system of coupled integral equations. Using asymptotic analysis, we separate the singular from the regular parts of the kernels and lead to the following system of singular integral equations in the case of distributed discrete defects 


$$
\begin{aligned}
-\sigma_{y y}^{c l . d .}(x-d, 0) & =\frac{\mu(3-2 \nu)}{2 \pi(1-\nu)} \int_{-a}^{a} \frac{B_{I}^{s d}(t)}{x-t} d t+\frac{\mu}{\pi a} \int_{-a}^{a} W^{s d}(t) \ln \frac{|x-t|}{\ell} d t \\
& +\frac{2 \mu}{\pi} \int_{-a}^{a} B_{I}^{s d}(t) R_{1}^{s d}(x-t) d t-\frac{\mu}{\pi a} \int_{-a}^{a} W^{s d}(t) R_{2}^{s d}(x-t) d t, \quad|x|<a \\
-m_{y z}^{c l . d .}(x-d, 0) & =-\frac{2 \mu \ell^{2}}{\pi a} \int_{-a}^{a} \frac{W^{s d}(t)}{x-t} d t+\frac{\mu}{\pi} \int_{-a}^{a} B_{I}^{s d}(t) \ln \frac{|x-t|}{\ell} d t \\
& -\frac{\mu}{\pi} \int_{-a}^{a} B_{I}^{s d}(t) R_{2}^{s d}(x-t) d t+\frac{\mu \ell}{2 \pi a} \int_{-a}^{a} W^{s d}(t) R_{3}^{s d}(x-t) d t, \quad|x|<a
\end{aligned}
$$

where the quantities $B_{I}^{s d}(t)$ and $W^{s d}(t)$ represent the densities of the climb dislocation and the constrained wedge disclination respectively. These are given as

$$
\begin{array}{ll}
B_{I}^{s d}(t)=\frac{d b_{y}(t)}{d t}=-\frac{d \Delta u_{y}(t)}{d t}, & \Delta u_{y}(x)=-\int_{-a}^{x} B_{I}^{s d}(t) d t, \\
W^{s d}(t)=a \frac{d \Omega_{z}(t)}{d t}=a \frac{d \Delta \omega(t)}{d t}, & \Delta \omega(x)=\frac{1}{a} \int_{-a}^{x} W^{s d}(t) d t .
\end{array}
$$

In Eqs. (40) and (41), $\Delta u_{y}(x)$ is the relative opening displacement and $\Delta \omega(x)$ the relative rotation between the upper and lower crack faces respectively. Therefore, in the case of discrete dislocations distribution, the climb dislocation density corresponds to the negative of the slope and the constrained disclination density to the curvature at any point along the crack faces. Also, the loading that appears on the left-hand side of Eqs. (38) and (39) is given in Eq. (25) for a discrete climb dislocation and Eq. (28) $)_{1}$ for a climb dislocation dipole.

Moreover, the regular kernels $R_{q}^{\text {sd }}(x-t)$ with $q=1,2,3$, have the following form

$$
R_{1}^{s d}(x-t)=\frac{1}{x-t}\left[\frac{2 \ell^{2}}{(x-t)^{2}}-K_{2}\left(\frac{|x-t|}{\ell}\right)-\frac{1}{2}\right]
$$




$$
\begin{aligned}
& R_{2}^{s d}(x-t)=\left[\frac{2 \ell^{2}}{(x-t)^{2}}-K_{2}\left(\frac{|x-t|}{\ell}\right)\right]+\left[K_{0}\left(\frac{|x-t|}{\ell}\right)+\ln \left(\frac{|x-t|}{\ell}\right)\right], \\
& R_{3}^{s d}(x-t)=\operatorname{sgn}(x-t) \cdot G_{1,3}^{2,1}\left(\frac{(x-t)^{2}}{4 \ell^{2}} \mid \begin{array}{c}
1 \\
-1 / 2,1 / 2,0
\end{array}\right)+\frac{4 \ell}{x-t} .
\end{aligned}
$$

Accordingly, following a displacement-based formulation, we obtain

$$
\begin{aligned}
& -\sigma_{y y}^{c l . d .}(x-d, 0)=\frac{\mu(3-2 \nu)}{2 \pi(1-\nu)} \text { F.P. } \int_{-a}^{a} \frac{B_{I}^{d d}(t)(t)}{(x-t)^{2}} d t-\frac{\mu}{\pi a} \int_{-a}^{a} \frac{W^{d d}(t)}{x-t} d t \\
& -\frac{\mu}{4 \pi \ell^{2}} \int_{-a}^{a} B_{I}^{d d}(t) \ln \frac{|x-t|}{\ell} d t+\frac{\mu}{\pi} \int_{-a}^{a} B_{I}^{d d}(t) R_{1}^{d d}(x-t) d t \\
& +\frac{\mu}{\pi a} \int_{-a}^{a} W^{d d}(t) R_{2}^{d d}(x-t) d t, \quad|x|<a, \\
& -m_{y z}^{c l . d .}(x-d, 0)=-\frac{2 \mu \ell^{2}}{\pi a} \text { F.P. } \int_{-a}^{a} \frac{W^{d d}(t)}{(x-t)^{2}} d t-\frac{\mu}{\pi} \int_{-a}^{a} \frac{B_{I}^{d d}(t)}{x-t} d t \\
& -\frac{\mu}{\pi a} \int_{-a}^{a} W^{d d}(t) \ln \frac{|x-t|}{\ell} d t+\frac{\mu}{\pi} \int_{-a}^{a} B_{I}^{d d}(t) R_{2}^{d d}(x-t) d t \\
& +\frac{\mu}{\pi a} \int_{-a}^{a} W^{d d}(t) R_{3}^{d d}(x-t) d t, \quad|x|<a,
\end{aligned}
$$

where the symbol F.P. $\int$ denotes a Hadamard finite-part integral (see e.g. [Kaya and Erdogan, 1987; Kutt, 1975; Monegato, 1994]). Now, the densities of climb dislocation dipoles and constrained wedge disclination dipoles, $B_{I}(t)$ and $W(t)$, are defined as

$$
B_{I}^{d d}=\frac{d b_{y y}(t)}{d t}=\Delta u_{y}(x), \quad W^{d d}(t)=-a \frac{d \Omega_{z y}(t)}{d t}=-a \Delta \omega(t)
$$

Hence, the climb dislocation dipole density represents the relative displacement while the constrained wedge disclination dipole density corresponds to the relative rotation of the crack 
faces at any point across the crack length. Further, the kernels $R_{q}^{d d}(x-t)$, for $q=1,2,3$, are now defined as

$$
\begin{aligned}
R_{1}^{d d}(x-t)= & \frac{6}{(x-t)^{2}}\left[\frac{2 \ell^{2}}{(x-t)^{2}}-K_{2}(|x-t| / \ell)-\frac{1}{6}\right] \\
& +\frac{1}{\ell^{2}}\left[K_{0}(|x-t| / \ell)-K_{2}(|x-t| / \ell)+\frac{1}{4} \ln (|x-t| / \ell)\right], \\
R_{2}^{d d}(x-t)= & -\frac{1}{x-t}\left[\frac{4 \ell^{2}}{(x-t)^{2}}-K_{2}(|x-t| / \ell)-1\right], \\
R_{3}^{d d}(x-t)= & \frac{2 \ell^{2}}{(x-t)^{2}}+K_{0}(|x-t| / \ell)-K_{2}(|x-t| / \ell)+\ln (|x-t| / \ell) .
\end{aligned}
$$

Employing asymptotic analysis, it can be shown that all kernels in Eq. (46) are regular as $x \rightarrow t$ and $\ell>0$.

An important difference between the two approaches is that in the slope formulation, the following closure conditions must be satisfied to ensure that the normal displacement and the rotation are single-valued

$$
\int_{-a}^{a} B_{I}^{s d}(t) d t=0, \quad \int_{-a}^{a} W^{s d}(t) d t=0
$$

The previous conditions ensure that $\Delta u_{y}(a)=\Delta u_{y}(-a)=0$ and $\Delta \omega(a)=\Delta \omega(-a)=0$, i.e. that there is no remaining net dislocation along the crack length. In the displacement-based formulation, the two dislocations that form the dipole cancel each other out (self-annihilation) and therefore, no extra closure conditions are required.

Next, the unknown defect densities should be expressed in a way to account for the asymptotic behavior of the displacement and the rotation the crack-tips. Huang et al. [1997] showed that in the context of couple-stress elasticity, both the displacement $u_{y}$ and the rotation $\omega$ behave as $r^{1 / 2}$ near the crack-tips, where $r$ is the radial distance from the crack-tip. Thus, the defect densities are written as 


$$
\begin{aligned}
& B_{I}^{s d}(\tilde{t})=\sum_{n=0}^{\infty} b_{n} T_{n}(\tilde{t})\left(1-\tilde{t}^{2}\right)^{-1 / 2}, \quad W^{s d}(\tilde{t})=\sum_{n=0}^{\infty} c_{n} T_{n}(\tilde{t})\left(1-\tilde{t}^{2}\right)^{-1 / 2}, \quad|\tilde{t}|<1, \\
& B_{I}^{d d}(\tilde{t})=\sum_{n=0}^{\infty} b_{n} U_{n}(\tilde{t})\left(1-\tilde{t}^{2}\right)^{1 / 2}, \quad W^{d d}(\tilde{t})=\sum_{n=0}^{\infty} c_{n} U_{n}(\tilde{t})\left(1-\tilde{t}^{2}\right)^{1 / 2}, \quad|\tilde{t}|<1,
\end{aligned}
$$

where $T_{n}(\tilde{t})$ and $U_{n}(\tilde{t})$ are the Chebyshev polynomials of the first and second kind respectively (see e.g. Abramowitz and Stegun [1964]), $\left(b_{n}, c_{n}\right)$ are unknown parameters and $\tilde{t}=t / a$.

Considering the above, the system of integral equations (Eqs. (38)-(39) or Eqs. (43)-(44) is solved numerically using an appropriate collocation technique. The regular integrals in these expressions are evaluated using the standard Gauss-Chebyshev quadrature whereas the hypersingular, singular, and weakly singular (logarithmic) integrals are calculated in closed form. It is noted that the convergence of the obtained solution is dependent on the ratio $\ell / a$.

\subsection{Antiplane mode interaction problem}

The corrective stresses for the antiplane shear mode problem (Eq. (35)) are produced by a continuous distribution of discrete screw dislocations or of infinitesimal screw dislocation dipoles along the crack faces. In the case of slope formulation, the elastic field generated is obtained by integrating the influence function (Eq. (36)) along the crack faces. With the use of asymptotic analysis, we separate the singular from the regular part of the kernel and obtain the following hyper-singular integral equation with cubic and Cauchy type singularities

$$
\begin{array}{r}
-t_{y z}^{\left(b_{z}\right)}(x-d, 0)=- \\
-c_{1} \ell^{2} \text { F.P. } \int_{-a}^{a} \frac{B_{I I I}^{s d}(t)}{(x-t)^{3}} d t+c_{2} \int_{-a}^{a} \frac{B_{I I I}^{s d}(t)}{x-t} d t \\
+c_{3} \int_{-a}^{a} B_{I I I}^{s d}(t) R_{4}^{s d}(x-t) d t, \quad|x|<a .
\end{array}
$$

The screw dislocation density at any point along the crack faces is given as 


$$
B_{I I I}^{s d}(t)=\frac{d b_{z}(t)}{d t}=-\frac{d \Delta w(t)}{d t}, \quad \Delta w(x)=-\int_{-a}^{x} B_{I I I}^{s d}(t) d t
$$

where $\Delta w=w\left(t, 0^{+}\right)-w\left(t, 0^{-}\right)$is the relative out-of-plane displacement between the upper and lower crack faces. Also, the constants $c_{i}$, with $i=1,2,3$, are written as

$$
c_{1}=\frac{\mu(1+\beta)(3-\beta)}{2 \pi}, \quad c_{2}=\frac{\mu\left(\beta^{2}+2 \beta+9\right)}{16 \pi}, \quad c_{3}=\frac{\mu(1+\beta)^{2}}{\pi},
$$

and the regular kernel $R_{4}^{s d}(x-t)$ as

$$
\begin{aligned}
R_{4}^{s d}(x-t)= & -\frac{\ell^{2}}{(x-t)^{3}}\left[6\left(K_{2}\left(\frac{|x-t|}{\ell}\right)-\frac{2 \ell^{2}}{(x-t)^{2}}\right)+\frac{1}{2}\right] \\
& +\frac{1}{4(x-t)}\left[3 K_{0}\left(\frac{|x-t|}{\ell}\right)-5 K_{2}\left(\frac{|x-t|}{\ell}\right)-\frac{1}{4}\right] .
\end{aligned}
$$

Further, employing a formulation based on defect dipoles, the integration of the influence function (Eq. (37)) along the crack faces leads to the following hyper-singular integral equation with fourth order, quadratic, and logarithmic singularities

$$
\begin{aligned}
-t_{y z}^{s c r . d .}( & (x-d, 0)=-3 c_{1} \ell^{2} \text { F.P. } \int_{-a}^{a} \frac{B_{I I I}^{d d}(t)}{(x-t)^{4}} d t+c_{2} \text { F.P. } \int_{-a}^{a} \frac{B_{I I I}^{d d}(t)}{(x-t)^{2}} d t \\
& -\frac{\mu}{32 \ell^{2}} \int_{-a}^{a} B_{I I I}^{d d}(t) \ln \frac{|x-t|}{\ell} d t+c_{3} \int_{-a}^{a} B_{I I I}^{d d}(t) R_{4}^{d d}(x-t) d t, \quad|x|<a,
\end{aligned}
$$

where the screw dislocation dipole density is given by the expression

$$
B_{I I I}^{d d}(t)=\frac{d b_{z y}(t)}{d t}=\Delta w(t)
$$


The constants $c_{i}$ in Eq. (54) are provided in Eq. (52) and the kernel $R_{4}^{d d}(x-t)$ now reads as

$$
\begin{aligned}
R_{4}^{d d}(x-t)= & -\frac{\ell^{2}}{(x-t)^{4}}\left[30\left(K_{2}(|x-t| / \ell)-\frac{2 \ell^{2}}{(x-t)^{2}}\right)+\frac{3}{2}\right] \\
& +\frac{1}{8(x-t)^{2}}\left[30 K_{0}(|x-t| / \ell)-54 K_{2}(|x-t| / \ell)-\frac{1}{2}\right] \\
& +\frac{1}{4 \ell^{2}}\left[K_{0}(|x-t| / \ell)-K_{2}(|x-t| / \ell)+\frac{1}{8} \ln (|x-t| / \ell)\right] .
\end{aligned}
$$

It is noted that for $\beta=-1$, Eqs. (50) and (54) reduce to the corresponding governing equations of classical elasticity.

Similar to the opening mode problem, the governing equation based on a distribution of discrete defects (Eq. (50)) is accompanied by the following closure condition to ensure uniqueness of the values of the antiplane displacement for a closed loop around the crack

$$
\int_{-a}^{a} B_{I I I}^{s d}(t) d t=0
$$

Moreover, in the context of couple-stress elasticity Zhang et al. [1998] employed the asymptotic Williams technique to show that the antiplane displacement $w$ behaves as $\sim r^{3 / 2}$ near the crack-tip region. Hence, the unknown defect densities may be written as

$$
\begin{array}{ll}
B_{I I I}^{s d}(\tilde{t})=\sum_{n=0}^{\infty} b_{n} U_{n}(\tilde{t})\left(1-\tilde{t}^{2}\right)^{1 / 2}, & |\tilde{t}|<1, \\
B_{I I I}^{d d}(\tilde{t})=\sum_{n=0}^{\infty} b_{n} U_{n}(\tilde{t})\left(1-\tilde{t}^{2}\right)^{3 / 2}, & |\tilde{t}|<1,
\end{array}
$$

where $U_{n}(\tilde{t})$ are the Chebyshev polynomials of the second kind and $b_{n}$ are unknown constants.

Finally, the singular integral equation (Eq. (50) or (54)) that governs the antiplane shear mode crack problem is solved numerically employing an appropriate collocation technique. 


\section{Evaluation of configurational forces}

\subsection{Opening mode interaction problem}

In this section, we derive the expressions for the energy release rate ( $J$-integral) in both cracktips and the Peach-Koehler force exerted on the defects. The J-integral in the context of couplestress elasticity was derived and proved path independent by Atkinson and Leppington [1974]; Atkinson and Leppington [1977]. A computationally efficient way to calculate the energy release rate was introduced by Freund [1972] and consists of using a rectangular shaped integration path that surrounds the (left or right) crack-tip and has vanishing height along the $y$-direction as $\varepsilon \rightarrow+0$ (Fig. 3). The advantage of this concept is that only the asymptotic near tip stress and displacement fields suffice for the evaluation of the $J$-integral.

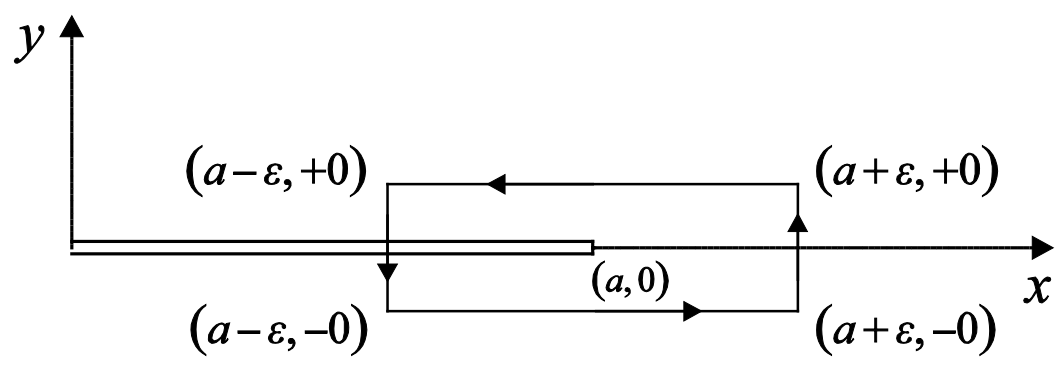

Fig. 3: Rectangular shaped contour for the calculation of $J$-integral around the right crack-tip.

Following this approach, the $J$-integral in the opening mode problem admits the following form

$$
J=-2 \lim _{\varepsilon \rightarrow+0}\left\{\int_{ \pm \alpha-\varepsilon}^{ \pm a+\varepsilon}\left[\sigma_{y y}\left(x, 0^{+}\right) \frac{\partial u_{y}\left(x, 0^{+}\right)}{\partial x}+m_{y z}\left(x, 0^{+}\right) \frac{\partial \omega\left(x, 0^{+}\right)}{\partial x}\right] d x\right\}
$$

Next, we employ asymptotic analysis to identify the near crack-tip behavior of the normal stress $\sigma_{y y}$, the couple-stress $m_{y z}$, and the gradients of displacement and rotation (Eqs. (38)-(41)). Based on these results, the $J$-integral at the right crack-tip is written under the form 


$$
\begin{gathered}
J_{r}=-2 a \lim _{\varepsilon \rightarrow 0}\left\{-\frac{\mu}{2}\left[\Lambda_{1}^{(r)}+\left(\frac{\ell}{a}\right)^{2} \Lambda_{2}^{(r)}\right] \int_{-\varepsilon / a}^{\varepsilon / a}\left(x_{+}\right)^{-1 / 2} \cdot\left(x_{-}\right)^{-1 / 2} d \bar{x}\right\} \\
=\frac{\mu \pi a}{2}\left[\Lambda_{1}^{(r)}+\left(\frac{\ell}{a}\right)^{2} \Lambda_{2}^{(r)}\right],
\end{gathered}
$$

where

$$
\Lambda_{1}^{(r)}=\frac{(3-2 \nu)}{4(1-\nu)}\left(\sum_{n=1}^{N} b_{n}\right)^{2}, \quad \Lambda_{2}^{(r)}=\left(\sum_{n=1}^{N} c_{n}\right)^{2}
$$

and $\bar{x}=\tilde{x}-1, \tilde{x}=x / a$. The integral in Eq. (61) is evaluated using Fisher's theorem for products of singular distributions [Fisher, 1971].

Due to the asymmetric nature of the loading in the interaction problems studied, the energy release rate will be different in the two crack-tips. Following a similar procedure, we obtain the following expression for the $J$-integral at the left crack-tip

$$
\begin{gathered}
J_{\ell}=-2 a \lim _{\varepsilon \rightarrow 0}\left\{\frac{\mu}{2}\left[\Lambda_{1}^{(\ell)}+\left(\frac{\ell}{a}\right)^{2} \Lambda_{2}^{(\ell)}\right] \int_{-\varepsilon / a}^{\varepsilon / a}\left(x_{+}\right)^{-1 / 2} \cdot\left(x_{-}\right)^{-1 / 2} d \bar{x}\right\} \\
=-\frac{\mu \pi a}{2}\left[\Lambda_{1}^{(\ell)}+\left(\frac{\ell}{a}\right)^{2} \Lambda_{2}^{(\ell)}\right],
\end{gathered}
$$

where

$$
\Lambda_{1}^{(\ell)}=\frac{(3-2 \nu)}{4(1-\nu)}\left(\sum_{n=1}^{N}(-1)^{n} b_{n}\right)^{2}, \quad \Lambda_{2}^{(\ell)}=\left(\sum_{n=1}^{N}(-1)^{n} c_{n}\right)^{2}
$$

Moreover, if we use the stress and kinematical expressions of the displacement-based formulation (Eqs. (43)-(45)), we lead to slightly different formulas. Specifically, the energy release rate in both crack-tips is still described by Eqs. (61) and (63) where now 


$$
\begin{aligned}
& \Lambda_{1}^{(r)}=\frac{(3-2 \nu)}{4(1-\nu)}\left(\sum_{n=0}^{N}(n+1) b_{n}\right)^{2}, \quad \Lambda_{2}^{(r)}=\left(\sum_{n=0}^{N}(n+1) c_{n}\right)^{2}, \\
& \Lambda_{1}^{(\ell)}=\frac{(3-2 \nu)}{4(1-\nu)}\left(\sum_{n=0}^{N}(-1)^{n}(n+1) b_{n}\right)^{2}, \quad \Lambda_{2}^{(\ell)}=\left(\sum_{n=0}^{N}(-1)^{n}(n+1) c_{n}\right)^{2} .
\end{aligned}
$$

In the framework of classical elasticity, the corresponding expressions for the $J$-integral can be obtained in closed form using the rectangular integration contour shown in Fig. 3 and the elastic fields of the problem [Markenscoff, 1993]. Indeed, the energy release rate at the right and left crack-tips due to the interaction with a discrete climb dislocation is given as

$$
\begin{gathered}
J_{r}^{\text {clas. }}=\frac{\mu b_{y}^{2}\left[1-\left(\frac{d+a}{d-a}\right)^{1 / 2}\right]\left[d-a-\left(d^{2}-a^{2}\right)^{1 / 2}\right]}{8 \pi a(1-\nu)(d-a)}, \\
J_{\ell}^{\text {clas. }}=-\frac{\mu b_{y}^{2}\left[1-\left(\frac{d+a}{d-a}\right)^{1 / 2}\right]\left[d-a-\left(d^{2}-a^{2}\right)^{1 / 2}\right]}{8 \pi a(1-\nu)(d+a)} .
\end{gathered}
$$

Similarly, the $J$-integral in both crack-tips due to the interaction with a climb dislocation dipole is written as

$$
J_{r}^{\text {clas. }}=\frac{\mu b_{y}^{2} w_{d}^{2}}{8 \pi a(1-\nu)\left(d^{2}-a\right)(d-a)^{2}}, \quad J_{\ell}^{\text {clas. }}=-\frac{\mu b_{y}^{2} w_{d}^{2}}{8 \pi a(1-\nu)\left(d^{2}-a\right)(d+a)^{2}}
$$

Accordingly, we evaluate the configurational Peach-Koehler force that is exerted on the discrete climb dislocation or on the climb dislocation dipole. To perform this calculation, it is convenient to consider a contour that surrounds both the crack and the defect (Fig. 4). Then, using the equilibrium relation between Peach-Koehler force and J-integral around a discrete dislocation [Eshelby, 1951], we may write the expressions

$$
F_{x}^{s d}=-\left(J_{r}+J_{\ell}\right), \quad F_{x}^{d d}=F_{x}^{s d 1}-F_{x}^{s d 2}=-\left(J_{r}+J_{\ell}\right)
$$


where $F_{x}^{s d}$ (or equivalently $J_{d}$ ) is the Peach-Koehler force along the $x$-direction exerted on the discrete climb dislocation, and $J_{r}$ and $J_{\ell}$ are the $J$-integral values at the right and left cracktip. In the case of the climb dislocation dipole, the total Peach-Koehler force in the $x$-direction, $F_{x}^{d d}$, is decomposed to the components $F_{x}^{s d 1}$ and $F_{x}^{s d 2}$ (or equivalently $J_{d 1}$ and $J_{d 2}$ ), which are the Peach-Koehler forces exerted on the closer and farther to the crack constituent dislocations.
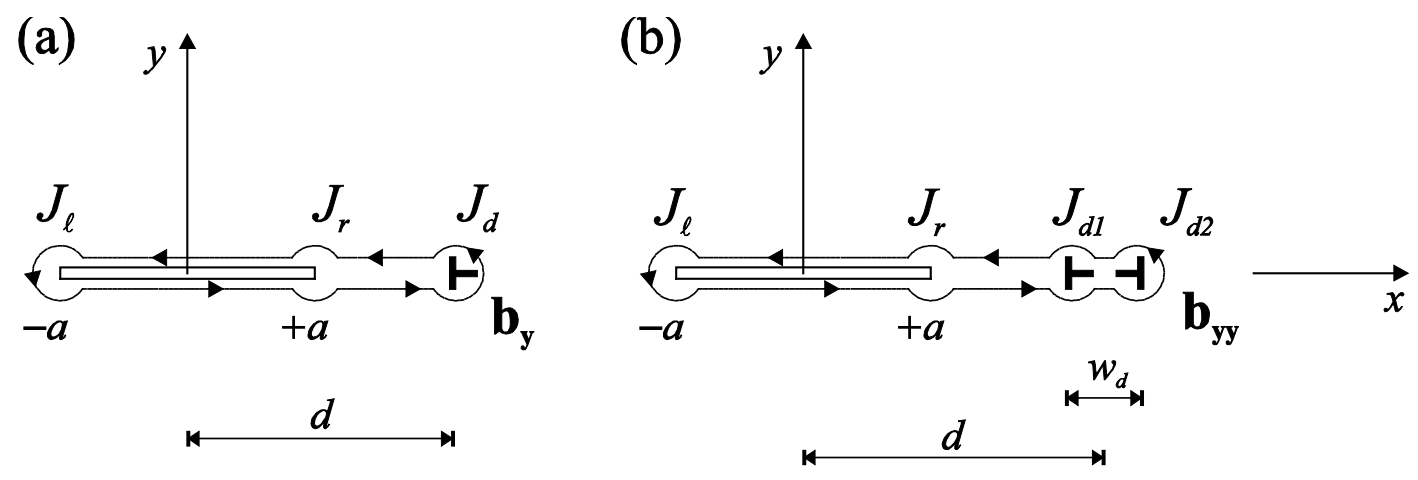

Fig. 4: Contours for the calculation of the Peach-Koehler force around a) a discrete climb dislocation and b) a climb dislocation dipole.

Finally, the corresponding expressions of the Peach-Koehler force for the two interaction problems in classical elasticity are given under the following forms

$$
F_{x}^{\text {sd,clas. }}=-\frac{\mu b_{y}^{2}}{2 \pi(1-\nu)} \frac{d-\left(d^{2}-a^{2}\right)^{1 / 2}}{d^{2}-a^{2}}, \quad F_{x}^{d d, \text { clas. }}=-\frac{\mu b_{y}^{2} w_{d}^{2} d}{2 \pi(1-\nu)\left(d^{2}-a\right)\left(d^{2}-a^{2}\right)^{2}}
$$

\subsection{Antiplane mode interaction problem}

Using the same integration contour, the $J$-integral in the antiplane mode problem admits the following form

$$
J=-2 \lim _{\varepsilon \rightarrow+0}\left\{\int_{ \pm a-\varepsilon}^{ \pm a+\varepsilon} t_{y z}\left(x, 0^{+}\right) \frac{\partial w\left(x, 0^{+}\right)}{\partial x} d x\right\}
$$


Using asymptotic analysis to identify the near crack-tip behavior of the total shear stress $t_{y z}$ and the gradient of antiplane displacement (Eqs. (50)-(51)), the $J$-integral at both crack-tips is given as

$$
\begin{aligned}
& J_{r}=-2 a \lim _{\varepsilon \rightarrow 0}\left\{\Lambda_{3}^{(r)} \int_{-\varepsilon / a}^{\varepsilon / a}\left(x_{+}\right)^{-3 / 2} \cdot\left(x_{-}\right)^{1 / 2} d \bar{x}\right\}=\pi a \Lambda_{3}^{(r)}, \\
& J_{\ell}=-2 a \lim _{\varepsilon \rightarrow 0}\left\{\Lambda_{3}^{(\ell)} \int_{-\varepsilon / a}^{\varepsilon / a}\left(x_{+}\right)^{1 / 2} \cdot\left(x_{-}\right)^{-3 / 2} d \bar{x}\right\}=-\pi a \Lambda_{3}^{(\ell)},
\end{aligned}
$$

where

$$
\Lambda_{3}^{(r)}=\frac{\pi c_{1} \ell^{2}}{8 a^{2}}\left(\sum_{n=1}^{N}(n+1) b_{n}\right)^{2}, \quad \Lambda_{3}^{(\ell)}=\frac{\pi c_{1} \ell^{2}}{8 a^{2}}\left(\sum_{n=1}^{N}(-1)^{n}(n+1) b_{n}\right)^{2}
$$

Alternatively, if a formulation based on screw dislocation dipoles is used (Eqs. (54)(55)), the energy release rate in both crack-tips is given by Eq. (72) where

$$
\Lambda_{3}^{(r)}=\frac{9 \pi c_{1} \ell^{2}}{8 a^{2}}\left(\sum_{n=0}^{N}(n+1) b_{n}\right)^{2}, \quad \Lambda_{3}^{(\ell)}=\frac{9 \pi c_{1} \ell^{2}}{8 a^{2}}\left(\sum_{n=0}^{N}(-1)^{n}(n+1) b_{n}\right)^{2}
$$

It is reminded that for the evaluation of the integral in Eq. (72), we employ Fisher's theorem for products of distributions of the bisection type.

Moreover, the $J$-integral value in classical elasticity is obtained in closed form using the rectangular shaped integration path described earlier and the elastic fields of the problem [Zhang and Li, 1991a]. The energy release rate at both crack-tips due to the interaction with a discrete screw dislocation is given as 


$$
\begin{gathered}
J_{r}=\frac{\mu b_{z}^{2}\left[1-\left(\frac{d+a}{d-a}\right)^{1 / 2}\right]\left[d-a-\left(d^{2}-a^{2}\right)^{1 / 2}\right]}{8 \pi a(d-a)}, \\
J_{\ell}=-\frac{\mu b_{z}^{2}\left[1-\left(\frac{d+a}{d-a}\right)^{1 / 2}\right]\left[d-a-\left(d^{2}-a^{2}\right)^{1 / 2}\right]}{8 \pi a(d+a)} .
\end{gathered}
$$

Accordingly, using the elastic solution for the interaction with a screw dislocation dipole [Lin et al., 1993], we obtain

$$
J_{r}^{\text {clas. }}=\frac{\mu b_{z}^{2} w_{d}^{2}}{8 \pi a\left(d^{2}-a\right)(d-a)^{2}}, \quad J_{\ell}^{\text {clas. }}=-\frac{\mu b_{z}^{2} w_{d}^{2}}{8 \pi a\left(d^{2}-a\right)(d+a)^{2}}
$$

\section{Results and discussion}

In this section, we present and discuss characteristic results of the four interaction problems. The main target of this analysis to emphasize on potential deviations from the classical elasticity theory when couple-stresses are considered.

Starting from the kinematical quantities, in Fig. 5 the effect of the ratio $a / \ell$ on the normal crack face displacement is shown for a discrete climb dislocation (Fig. 5a) and a climb dislocation dipole (Fig. 5b). Both defects are placed at the same distance from the crack-center, $d / a=2.5$, in a couple-stress material with Poisson's ratio $\nu=0.3$. A common observation for the two problems is that as the characteristic length becomes comparable to the crack halflength, the displacements decrease, i.e. the material exhibits a stiffer behavior. The classical elasticity solution (denoted by a dashed line) serves as an upper bound for all couple-stress elasticity displacement profiles. Besides, the crack is deformed in an asymmetric manner due to the nature of the applied loading. This effect is more pronounced when the defects are placed closer to the crack-tip. It is also noted that in the interaction with a climb dislocation dipole the produced fields are smaller in magnitude compared to the discrete defect interaction problem. 
However, the crack profile appears quantitively more distorted due to the presence of the crystal defect.
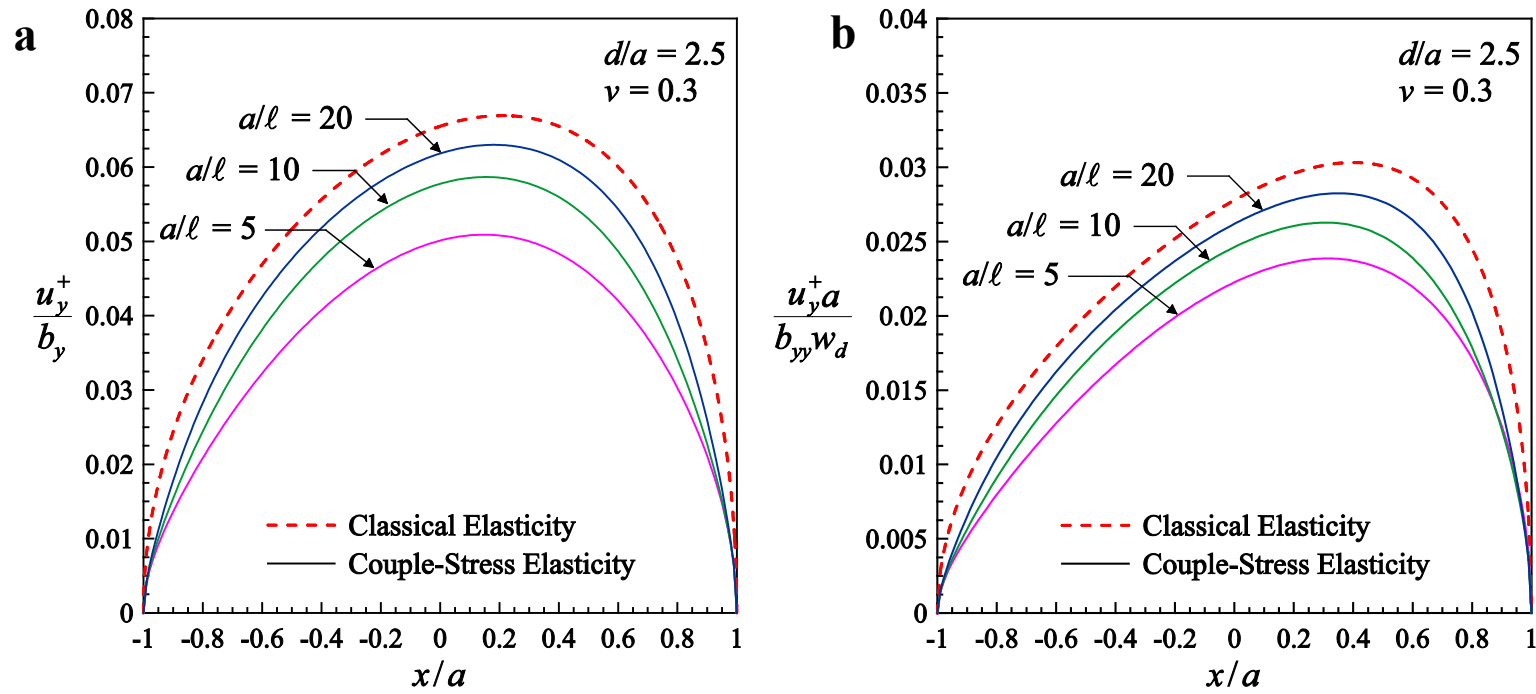

Fig. 5: Normalized upper-half crack displacement due to the interaction with a) a discrete climb dislocation and $\mathbf{b}$ ) a climb dislocation dipole lying at $d / a=2.5$ in a material with $\nu=0.3$.
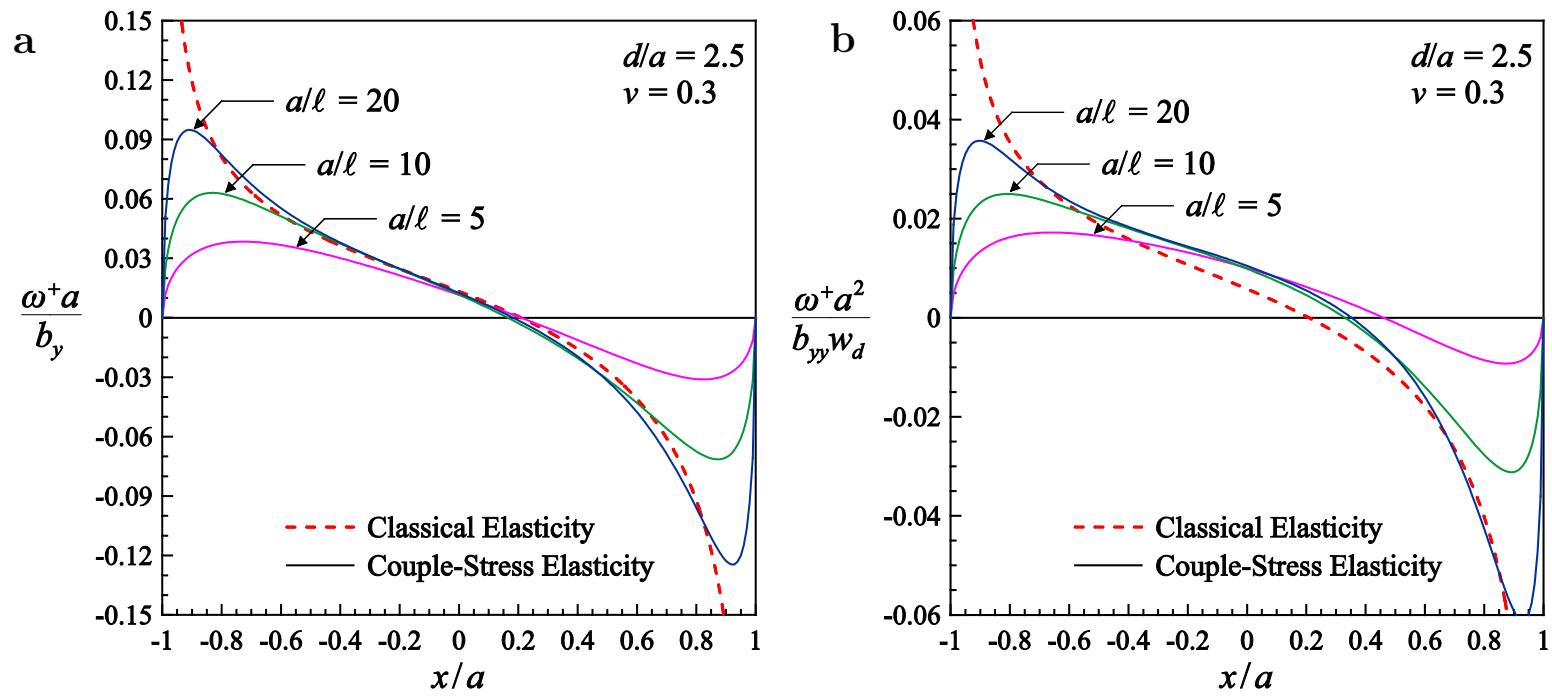

Fig. 6: Rotation profiles for various ratios $a / \ell$ due to the interaction with a) a discrete climb dislocation dipole and $\mathbf{b}$ ) a climb dislocation dipole lying at $d / a=2.5$ in a material with $\nu=0.3$.

Furthermore, we calculate the upper-half crack rotation for the same configuration for both interaction problems. The variation of these field with respect to the ratio $a / \ell$ is plotted in Fig. 6. The magnitude of the rotation is still higher for the same ratio $a / \ell$ in the interaction 
with a discrete defect, however, the extent of positive rotation across the crack length is larger in the interaction with a dislocation dipole $(-1 \leq x / a \leq 0.4)$. Also, the maximum in absolute value rotation is met near the right crack-tip as expected. Comparing the couple-stress solution to the classical elasticity one, it is noted that the incorporation of couple-stresses yields bounded results, which in fact tend to zero in both crack-tips. On the other hand, the classical elasticity solution (dashed line) exhibits a square-root singularity at the crack-tips. It is further observed that as $\ell \rightarrow 0$, the rotation profile in couple-stress elasticity becomes pointwise convergent to the classical elasticity unbounded solution.

Next, we study the variation of the stress intensity factor (SIF) in couple-stress theory at both crack-tips for the same interaction problems $(d / a=2.5)$. The SIF is defined at the right crack-tip as $K_{I}=\lim _{x \rightarrow a^{+}}[2 \pi(x-a)]^{1 / 2} \sigma_{y y}(x, 0)$. In Fig. 7 , the variation of the ratio $K_{I} / K_{I}^{\text {clas. }}$ at both crack-tips with respect to the ratio $\ell / a$ and the Poisson's ratio $\nu$ is given. We notice that the general trend of the SIFs ratio is comparable between the two problems, however, the response is significantly different between the two crack-tips, due to the asymmetry of the applied loading. First, when no couple-stress effects are considered $(\ell / a=0)$ the above ratio does not approach unity, as perhaps expected but instead exhibits a finite jump discontinuity. Sternberg and Muki [1967] attributed this behavior to the boundary layer effects that arise in couple-stress elasticity in singular stress-concentration problems. Remarkably, the value of this discontinuity is constant in both interaction problems and at both crack-tips. Then, as the ratio $\ell / a$ increases, the right crack-tip curves (continuous lines) monotonically increase in the range $0 \leq \ell / a \leq 1$. In the interaction with a climb dislocation dipole, the SIFs ratio decreases for larger values of $\ell / a$. On the other hand, the left crack-tip response (dashed line) is characterized by an initial decreasing branch which is followed by a monotonically increasing behavior. Interestingly enough, all curves approach asymptotically the value $(3-2 \nu)$ as $\ell / a \rightarrow \infty$. Also, in all cases, the SIF in couple-stress theory is significantly higher than the classical elasticity solution for any ratio $\ell / a$ (stress aggravation effect). 

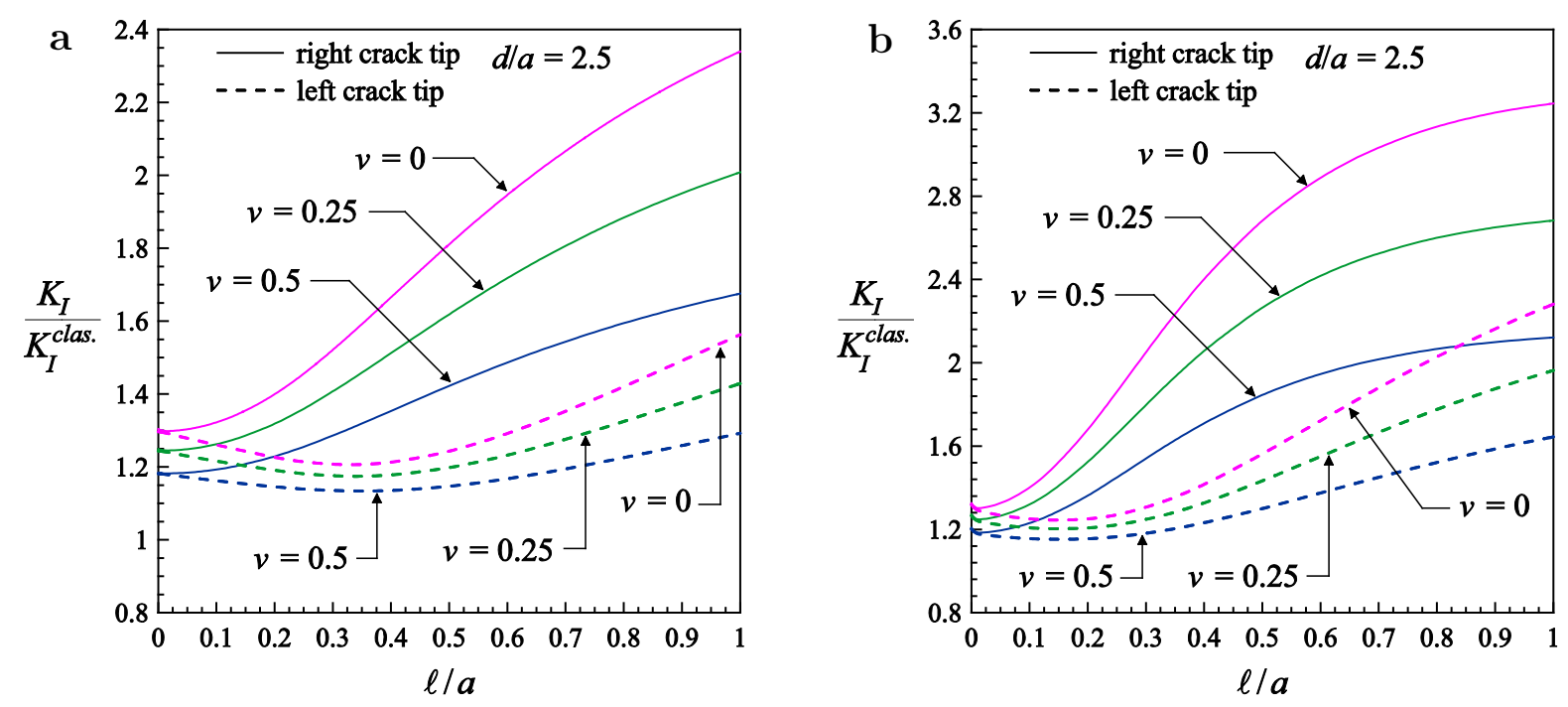

Fig. 7: a) Variation of the ratio of SIFs $K_{I} / K_{I}^{\text {clas. }}$ in couple-stress theory and in classical elasticity with respect to $\ell / a$ for $\mathbf{a}$ ) a discrete climb dislocation and b) a climb dislocation dipole lying at

$$
d / a=2.5
$$

Moreover, we evaluate numerically the energy release rate and study its dependence on the material and geometrical parameters of the problem. In Fig. 8, the variation of the ratio $J / J^{\text {clas. }}$ with respect to the ratio $\ell / a$ and the Poisson's ratio $\nu$ is plotted. Contrary to the SIFs response, the $J$-integral in couple-stress theory tends to the corresponding results of classical elasticity as the ratio $\ell / a \rightarrow 0$. Thereafter, the response is similar at the two crack-tips for both interaction problems. Specifically, as $\ell / a$ increases, the ratio initially reduces $\left(J<J^{\text {clas. }}\right)$ and reaches a minimum value. The exact $\ell / a$ of that minimum varies depending on the Poisson's ratio and the defect distance from the crack center $d / a$. Thereafter, a monotonically increasing behavior is observed $\left(J>J^{\text {clas. }}\right)$ for the interaction with a discrete dislocation (Fig. 8a). On the other hand, in the case of a dislocation dipole (Fig. 8b) the ratio $J / J^{\text {clas. }}$ decreases for $\ell / a>1$. Eventually, all curves tend asymptotically to the same value $(3-2 \nu)$ as $\ell / a \rightarrow \infty$. Hence, for small values of $\ell / a$, the energy release rate is lower than the classical elasticity value revealing a strengthening effect when couple-stresses are taken into account whereas for higher values of the ratio $\ell / a$ a weakening effect is noticed since $J / J^{\text {clas. }}>1$. 

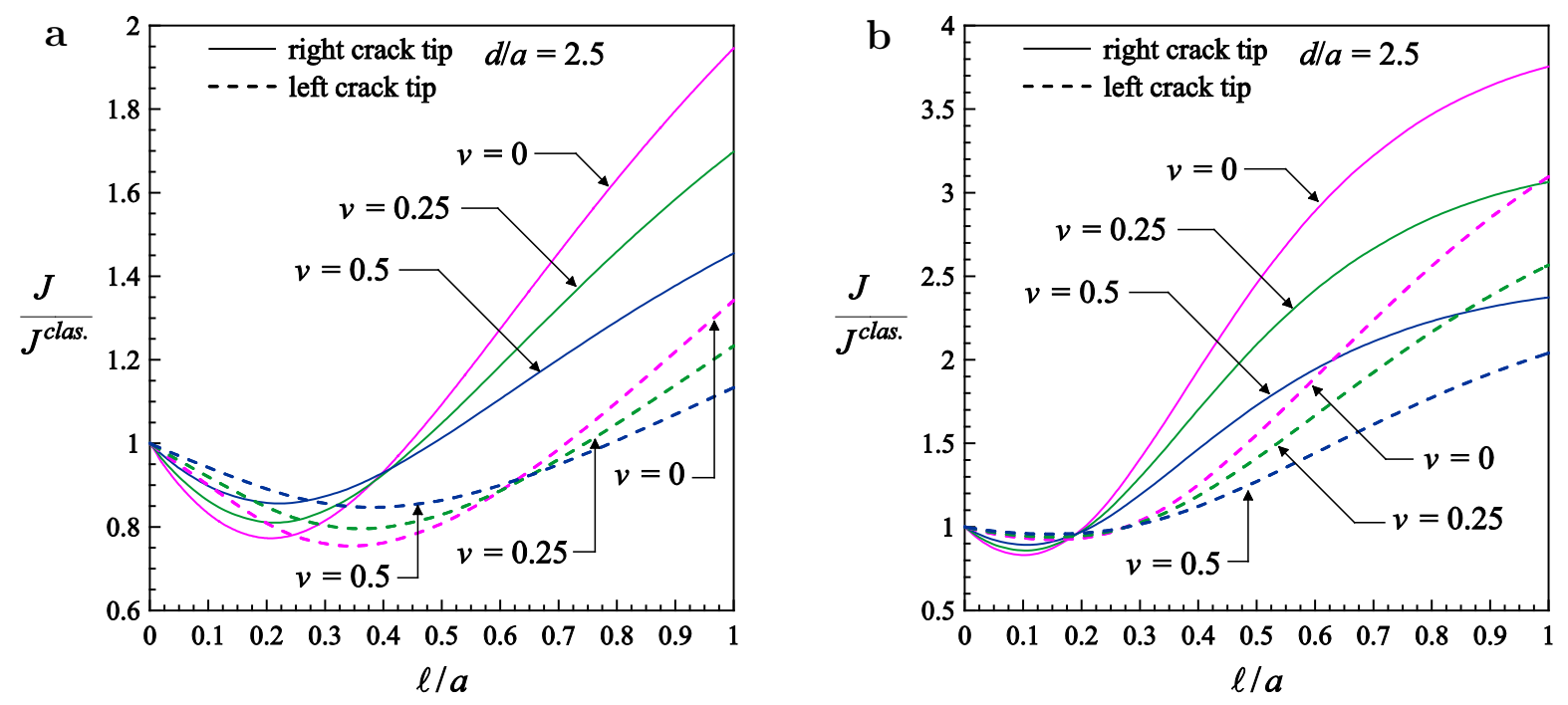

Fig. 8: a) Variation of the ratio of $J$-integrals $\left(J / J^{\text {clas. }}\right)$ in couple-stress theory and in classical elasticity with respect to $\ell / a$ for $\mathbf{a}$ ) a discrete climb dislocation and b) a climb dislocation dipole lying at $d / a=2.5$.
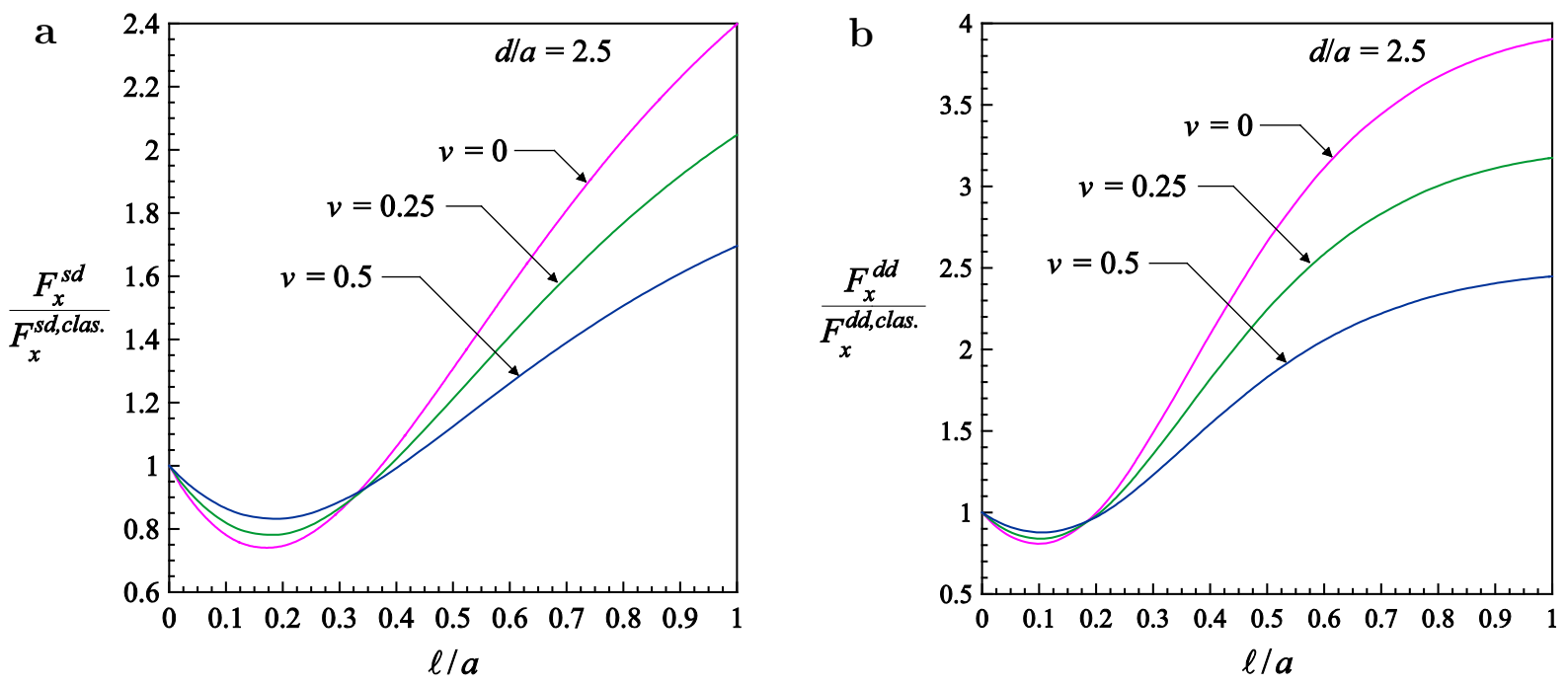

Fig. 9: Variation of the ratio of the Peach-Koehler forces with respect to $\ell / a$ for a) a discrete climb dislocation and $\mathbf{b}$ ) a climb dislocation dipole lying at $d / a=2.5$.

Based on the energy release rate results, we anticipate the configurational force exerted on either defect to exhibit a similar behavior. In Fig. 9 , the dependence of the ratio $F_{x} / F_{x}^{\text {clas. }}$ (i.e. $F_{x}^{s d} / F_{x}^{s d, c l a s .}$ for the discrete dislocation and $F_{x}^{d d} / F_{x}^{d d, c l a s .}$ for the dislocation dipole) on the ratio $\ell / a$ and the Poisson's ratio $\nu$ is shown. Both defects are placed at the same distance, $d / a=2.5$. We observe that for $\ell / a \rightarrow 0$ there is a smooth transition to classical elasticity as 
the Peach-Kohler force tends to its classical elasticity counterpart. As the ratio $\ell / a$ increases, the ratio $F_{x} / F_{x}^{\text {clas. }}$ decreases until a minimum value in the range $0.1 \leq \ell / a \leq 0.2$ and then increases monotonically. In the case of a climb dislocation dipole, the ratio decreases for $\ell / a>1$ and again all curves approach the asymptotic value $(3-2 \nu)$ as $\ell / a \rightarrow \infty$. Overall, the defect configurational force is higher than the classical elasticity solution for a large range of values of the ratio $\ell / a$, predicting thus a weakening effect for the material under consideration.

Next, we present some results for the antiplane interaction problems. The effect of the ratio $a / \ell$ on the antiplane displacement $w$ is shown in Fig. 10 considering that both the discrete screw dislocation and the screw dislocation dipole lie at a distance $d / a=2.5$ from the crackcenter in a couple-stress material with $\beta=0$. The conclusions drawn for the opening mode problems still hold here. Indeed, the material exhibits a stiffer behavior as the crack length becomes comparable to the characteristic length $\ell$ and the classical elasticity solution (dashed line) is an upper bound for couple-stress elasticity. More importantly, focusing on the inset of these figures, we notice that the crack faces close in a smoother way $\left(\bar{x}^{3 / 2}\right)$ compared to the classical elasticity prediction $\left(\sim \bar{x}^{1 / 2}\right)$. This argument is also verified through asymptotic analysis. The generated displacements are smaller in magnitude in the dipole interaction problem, which is expected since the stress field produced by this defect is generally weaker compared to the discrete dislocation case.

Further, the distribution of the total shear stress $t_{y z}$ is plotted in Fig. 11 for the same geometrical configuration $(d / a=2.5)$ in a body with $a / \ell=500$ and three values of the parameter $\beta$. The distance is measured from the right crack-tip in the $x$-axis where a new variable $\bar{x}=x-a$ is introduced for convenience. It is noted that the stress values are higher in the discrete dislocation case as expected. Also, the obtained response when couple-stresses are considered is considerably different to the classical elasticity solution (dashed line). The total shear stress admits negative values in a small region $(\bar{x}<0.5 \ell)$ ahead of both crack-tips and therefore shows a cohesive-traction character along the prospective fracture zone. The width of this zone is reduced as $\beta \rightarrow-1$ whereas the maximum value of the total shear stress increases. 
Also, a bounded minimum is observed for $\bar{x}<2 \ell$ while for $\bar{x}>2 \ell$ the classical elasticity solution is recovered.
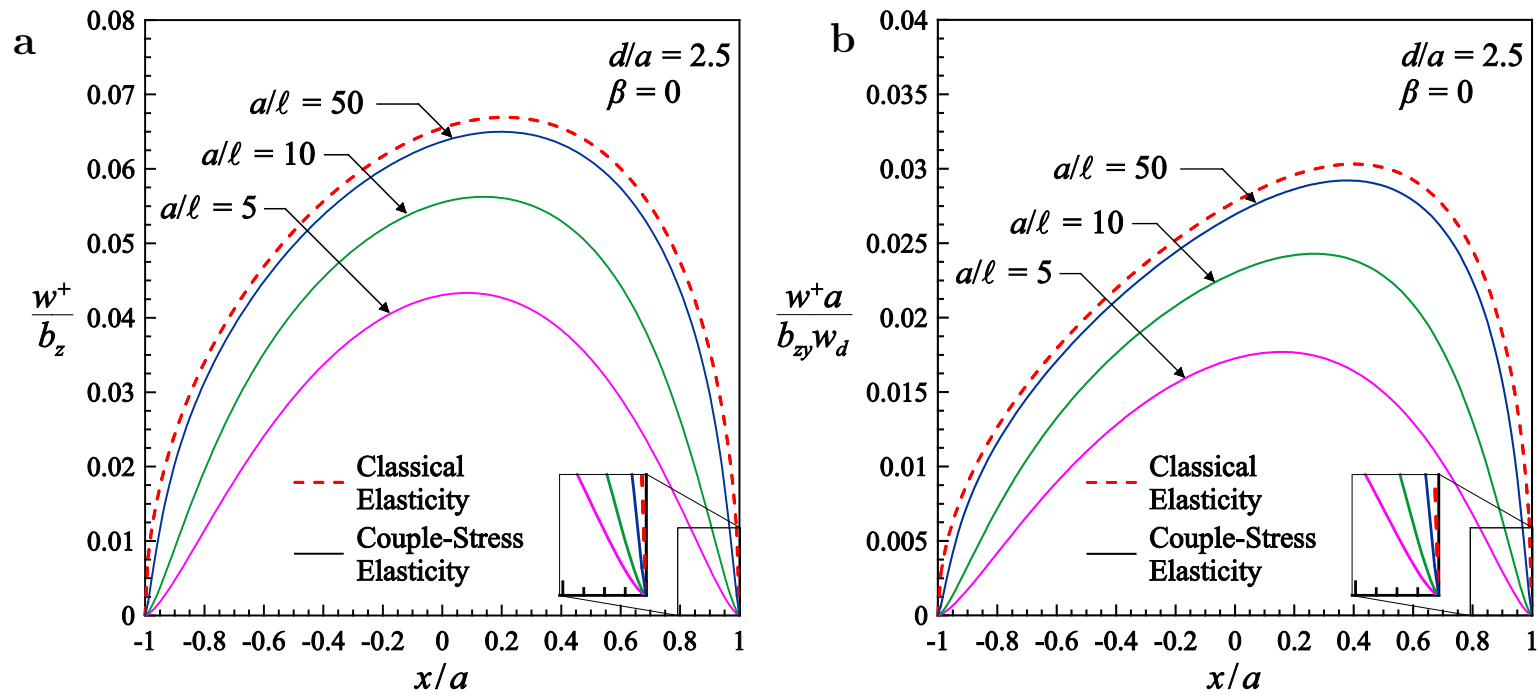

Fig. 10: Normalized upper-half crack antiplane displacement profile for various ratios $a / \ell$ due to the interaction with a) a discrete screw dislocation and $\mathbf{b}$ ) a screw dislocation dipole lying at $d / a=2.5$ in a material with $\beta=0$.
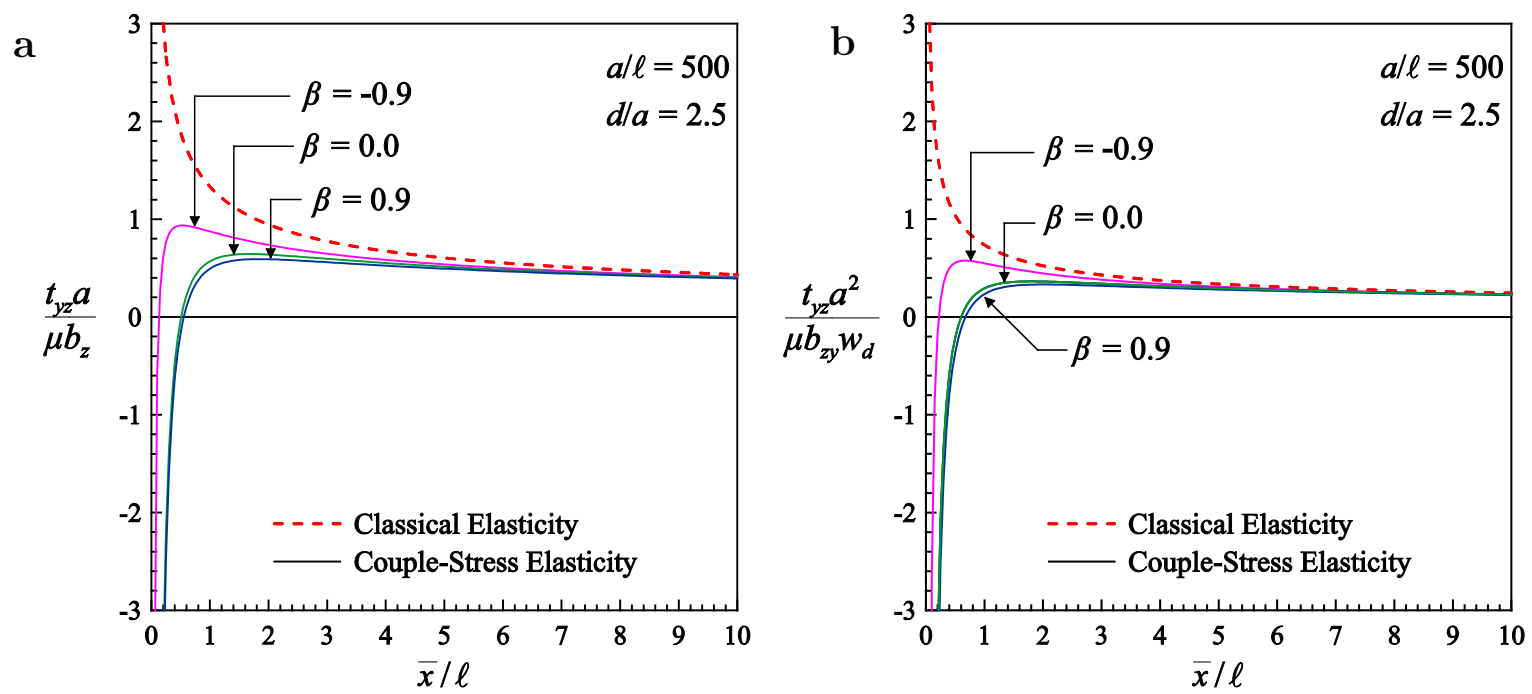

Fig. 11: Variation of the total shear stress $t_{y z}$ ahead of the right crack-tip due to the interaction with a) a discrete screw dislocation and $\mathbf{b}$ ) a screw dislocation dipole lying at $d / a=2.5$ in a medium with $a / \ell=500$ for different values of $\beta$. 
Then, we calculate the energy release rate at both crack-tips based on the analysis presented in Section 5.2. The variation of the ratio $J / J^{\text {clas. }}$ with respect to the ratio $\ell / a$ and the parameter $\beta$ for both defects is given in Fig. 12. The only similarity of the obtained response to the opening mode problems is that the ratio $J / J^{\text {clas. }}$ tends to unity as $\ell / a \rightarrow 0$. However, in the antiplane mode problems the ratio of $J$-integrals monotonically decreases as $\ell / a$ increases revealing therefore a strengthening effect when couple-stresses are introduced. This behavior is independent of the position of either crystal defect. Further, the ratio $J / J^{\text {clas. }}$ is always higher at the left crack-tip, contrary to the opening mode problem. It is also interesting to remark that the ratio $J / J^{\text {clas. }}$ tends to zero for $\beta \geq 0$ and $\ell / a>0.60$, which is attributed to the nature of the loading generated by the antiplane defects.
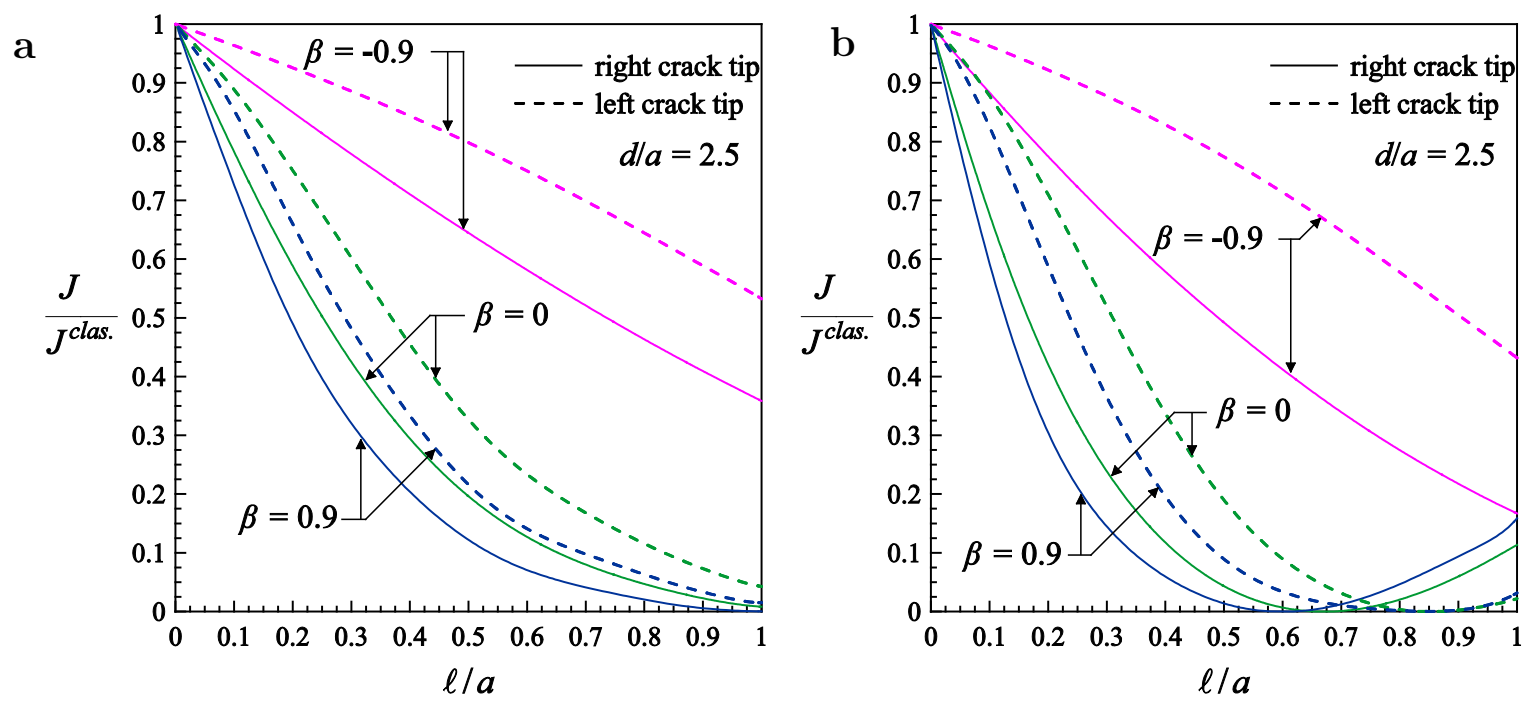

Fig. 12: Variation of the ratio of $J$-integrals in couple-stress theory and in classical elasticity with $\ell / a$ for $\mathbf{a}$ ) a discrete screw dislocation and $\mathbf{b}$ ) a screw dislocation dipole lying at $d / a=2.5$.

\section{Concluding remarks}

In the present study, interaction problems between finite-length cracks and crystal defects were investigated in the framework of couple-stress elasticity. The crack problems were formulated based on the distributed dislocation technique. In this context, different strain nuclei were used so that the governing equations were expressed based on the displacement gradient or the 
displacement itself. In view of the above, two sets of problems were analysed: the opening mode that corresponds to the interaction with climb defects and the antiplane mode where screw dislocation were considered. All problems were solved numerically while the expressions of the configurational forces acting on the defects were derived in the context of classical elasticity.

Several novel results were obtained in this investigation. The cracked solid was found to behave in a more rigid way in all cases compared to the classical elasticity prediction. The produced stress and couple-stress fields were always higher when a single dislocation was considered to interact with the crack. These fields remain unbounded at the tip of the defects just as in classical elasticity theory. Further, the energy release rate is significantly influenced by the defect distance and the magnitude of the characteristic material length with respect to the crack length. In the opening mode problem, the energy release rate reveals either strengthening or weakening effects depending on the material parameters and the geometry whereas in the antiplane case, the energy release rate is always decreasing, revealing, thus, a strengthening effect when couple-stresses are introduced. These conclusions were also verified by evaluating the Peach-Koehler forces exerted on the defects. 


\section{References}

Abramowitz M and Stegun IA [1964] Handbook of mathematical functions (National Bureau of Standards, Applied Mathematics Series 55,

Atkinson C and Leppington FG [1974] "Some calculations of the energy-release rate G for cracks in micropolar and couple-stress elastic media," International Journal of Fracture 10, 599602.

Atkinson C and Leppington FG [1977] "The effect of couple stresses on the tip of a crack," International Journal of Solids and Structures 13, 1103-1122.

Ballarini R and Denda M [1988] "The interaction between a crack and a dislocation dipole," International Journal of Fracture 37, 61-71.

Baxevanakis KP, Gourgiotis PA and Georgiadis HG [2017a] "Interaction of cracks with dislocations in couple-stress elasticity. Part I: Opening mode," International Journal of Solids and Structures 118-119, 179-191.

Baxevanakis KP, Gourgiotis PA and Georgiadis HG [2017b] "Interaction of cracks with dislocations in couple-stress elasticity. Part II: Shear modes," International Journal of Solids and Structures 118-119, 192-203.

Bilby BA and Eshelby JD [1968] Dislocations and the theory of fracture. In: Liebowitz H (ed) Fracture, Vol. 1. New York: Academic Press.

Chan Y-S, Paulino GH and Fannjiang AC [2001] "The crack problem for nonhomogeneous materials under antiplane shear loading - a displacement based formulation," International Journal of Solids and Structures 38, 2989-3005.

Comninou M [1987] "The interaction between a dislocation and a crack: Closure considerations," Mechanics Research Communications 14, 245-253.

Cosserat E and Cosserat F [1909] Théorie des corps déformables (Hermann et Fils, Paris).

Dai DN [2002] "Modelling cracks in finite bodies by distributed dislocation dipoles," Fatigue and Fracture of Engineering Materials and Structures 25, 27-39.

Erdélyi A, Magnus W, Oberhettinger F, et al. [1953] Higher transcendental functions (McGrawHill, New York). 
Eshelby JD [1951] "The force on an elastic singularity," Philosophical Transactions of the Royal Society of London. Series A, Mathematical and Physical Sciences 244, 87-112.

Fisher B [1971] "The product of distributions," Quarterly Journal of Mathematics 22, 291-298.

Freund LB [1972] "Energy flux into the tip of an extending crack in an elastic solid," Journal of Elasticity 2, 341-349.

Gilman J [1964] "Influence of dislocation dipoles on physical properties," Discussions of the Faraday Society 38, 123-137.

Gourgiotis PA and Georgiadis HG [2008] "An approach based on distributed dislocations and disclinations for crack problems in couple-stress elasticity," International Journal of Solids and Structures 45, 5521-5539.

Hills DA, Kelly PA, Dai DN, et al. [1996] Solution of crack problems: the distributed dislocation technique (Kluwer Academic Publishers, Dordrecht).

Huang M, Rivera Díaz del Castillo PEJ and Li Z [2006] "Edge dislocation dipole emission from a blunt crack tip and its morphological effects," Scripta Materialia 54, 649-653.

Huang Y, Zhang L, Guo TF, et al. [1997] "Mixed mode near-tip fields for cracks in materials with strain-gradient effects," Journal of the Mechanics and Physics of Solids 45, 439465.

Kaya AC and Erdogan F [1987] "On the solution of integral equations with strongly strongly singular kernels," Quarterly of Applied Mathematics 45, 105-122.

Kobayashi S and Ohr SM [1980] "In situ fracture experiments in b.c.c. metals," Philosophical Magazine A 42, 763-772.

Koiter W. (1964) Couple stresses in the theory of elasticity. Parts I and II. Nederl. Akad. Wetensch. Proc. Ser. B. 17-29.

Korsunsky A and Hills D [1995] "The solution of plane crack problems by dislocation dipole procedures," Journal of Strain Analysis for Engineering Design 30, 21-27.

Korsunsky A and Hills D [1996] "The solution of crack problems by using distributed strain nuclei," Proceedings of the Institution of Mechanical Engineers, Part C: Journal of Mechanical Engineering Science 210, 23-31.

Kroupa F [1965] "Dislocation dipoles of infinitesimal width," Cechoslovackij fiziceskij zurnal B 15, 896-900. 
Kutt HR [1975] "The numerical evaluation of principal value integrals by finite-part integration," Numerische Mathematik 24, 205-210.

Lin KM, Hu CT and Lee S [1993] "Screw dislocation dipoles near an internal crack," Engineering Fracture Mechanics 45, 321-331.

Majumdar BS and Burns SJ [1981] "Crack tip shielding - an elastic theory of dislocations and dislocation arrays near a sharp crack," Acta Metallurgica 29, 579-588.

Markenscoff X [1993] "Interaction of dislocations and dislocation dipoles with cracks and anticracks," Materials Science Forum 123, 525-530.

Mindlin RD [1963] "Influence of couple-stresses on stress concentrations," Experimental Mechanics 3, 1-7.

Mindlin RD [1964] "Micro-structure in linear elasticity," Archive for Rational Mechanics and Analysis 16, 51-78.

Mindlin RD and Tiersten HF [1962] "Effects of couple-stresses in linear elasticity," Archive for Rational Mechanics and Analysis 11, 415-448.

Monegato G [1994] "Numerical evaluation of hypersingular integrals," Journal of Computational and Applied Mathematics 50, 9-31.

Mousavi SM [2016] "Dislocation-based fracture mechanics within nonlocal and gradient elasticity of bi-Helmholtz type - Part I: Antiplane analysis," International Journal of Solids and Structures 87, 222-235.

Mousavi SM and Lazar M [2015] "Distributed dislocation technique for cracks based on nonsingular dislocations in nonlocal elasticity of Helmholtz type," Engineering Fracture Mechanics 136, 79-95.

Rice JR and Thomson R [1974] "Ductile versus brittle behaviour of crystals," Philosophical Magazine 29, 73-97.

Sternberg E and Muki R [1967] "The effect of couple-stresses on the stress concentration around a crack," International Journal of Solids and Structures 3, 69-95.

Thomson R [1978] "Brittle fracture in a ductile material with application to hydrogen embrittlement," Journal of Materials Science 13, 128-142.

Thomson RM [1987] "Physics of fracture," Journal of Physics and Chemistry of Solids 48, 965983. 
Toupin RA [1962] "Elastic materials with couple-stresses," Archive for Rational Mechanics and Analysis 11, 385-414.

Wang S-D and Lee S [1992] "Mechanical equilibrium of an edge dislocation dipole near a crack tip," International Journal of Fracture 57, 317-324.

Wang S-D and Lee S [1993] "Edge dislocation dipoles near a semi-infinite crack tip," Engineering Fracture Mechanics 46, 297-309.

Weertman J [1978] "Fracture mechanics: A unified view for Griffith-Irwin-Orowan cracks," Acta Metallurgica 26, 1731-1738.

Weertman J [1996] Dislocation based Fracture Mechanics (World Scientific, Singapore).

Zhang L, Huang Y, Chen JY, et al. [1998] "The mode III full-field solution in elastic materials with strain gradient effects," International Journal of Fracture 92, 325-348.

Zhang T-Y and Li JCM [1991a] "Image forces and shielding effects of a screw dislocation near a finite-length crack," Materials Science and Engineering A 142, 35-39.

Zhang T-Y and Li JCM [1991b] "Image forces and shielding effects of an edge dislocation near a finite length crack," Acta Metallurgica et Materialia 39, 2739-2744. 